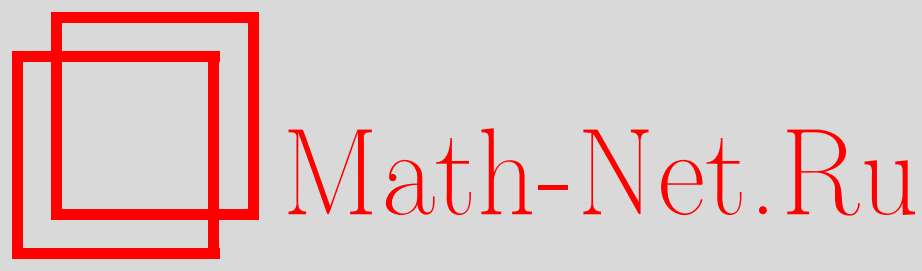

V. Baláž, J. Fialová, V. Grozdanov, S. Stoilova, O. Strauch, Hilbert Space with Reproducing Kernel and Uniform Distribution Preserving Maps. I, Совр. пробл. матем., 2013, выпуск 17, 30-60

DOI: https://doi.org/10.4213/spm42

Использование Общероссийского математического портала Math-Net.Ru подразумевает, что вы прочитали и согласны с пользовательским соглашением http://www . mathnet.ru/rus/agreement

Параметры загрузки:

IP : 54.164 .48 .24

26 апреля 2023 г., 17:46:31

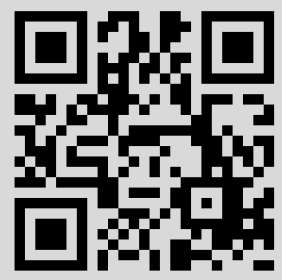




\title{
Hilbert Space with Reproducing Kernel and Uniform Distribution Preserving Maps. I
}

\author{
V. Baláž ${ }^{a}$, J. Fialová ${ }^{b}, V$. Grozdanov $^{c}$, S. Stoilova ${ }^{d}$, O. Strauch ${ }^{b}$ \\ ${ }^{a}$ Institute of Information Engineering Automation and Mathematics, Slovak University of Technology \\ ${ }^{b}$ Mathematical Institute, Slovak Academy of Sciences \\ ${ }^{c}$ South-West University "Neofit Rilski", Bulgaria \\ ${ }^{d}$ Institute of Mathematics and Informatics, Bulgarian Academy of Sciences
}

Dedicated to the memory of Anatolii Alekseevich Karatsuba

\section{Introduction}

A map $\Phi:[0,1]^{s} \rightarrow[0,1]^{s}$ is called uniform distribution preserving (u.d.p.) map if for every uniformly distributed (u.d.) sequence $\mathbf{x}_{n}, n=1,2, \ldots$, the image $\Phi\left(\mathbf{x}_{n}\right)$ is again u.d. Let $H$ be a Hilbert space of real functions $f(\mathbf{x}), \mathbf{x} \in[0,1)^{s}$, with a reproducing kernel $K(\mathbf{x}, \mathbf{y})$, and let $\mathbf{x}_{0}, \ldots, \mathbf{x}_{N-1} \in[0,1)^{s}$ be an $N$-terms sequence. In the quasi-Monte Carlo (QMC) integration the following mean square worst-case error

$$
\int_{[0,1]^{s}} \sup _{\substack{f \in H \\\|f\| \leqslant 1}}\left|\frac{1}{N} \sum_{n=0}^{N-1} f\left(\mathbf{x}_{n} \oplus \boldsymbol{\sigma}\right)-\int_{[0,1]^{s}} f(\mathbf{x}) d \mathbf{x}\right|^{2} d \boldsymbol{\sigma}
$$

is studied for digital shift $\mathbf{x} \oplus \boldsymbol{\sigma} .{ }^{1}$ This quantity is the mean value of the $L^{2}$ discrepancy in QMC integration in a Hilbert space, see Section 5. For some u.d.p. $\Phi(\mathbf{x})$ the following mean square worst-case error

$$
\int_{[0,1]^{s}} \sup _{\substack{f \in H \\\|f\| \leqslant 1}}\left|\frac{1}{N} \sum_{n=0}^{N-1} f\left(\Phi\left(\mathbf{x}_{n} \oplus \boldsymbol{\sigma}\right)\right)-\int_{[0,1]^{s}} f(\mathbf{x}) d \mathbf{x}\right|^{2} d \boldsymbol{\sigma}
$$

is less than (1). For example, Cristea, Dick, Leobacher and Pillichshammer [1] consider a Sobolev space of functions for which the second partial derivatives are square integrable, van der Corput sequence $x_{n}, n=0,1, \ldots, N-1$, with base $b=2$ and $N=2^{\beta}$ and the u.d.p. $\operatorname{map}^{2} \Phi_{0}(x)=$ $1-|2 x-1|$. They found (1) as $O\left(1 / N^{2}\right)$ and (2) as $O\left(1 / N^{4}\right) \cdot{ }^{3}$ A similar result for $x_{n}=n / N$ and $x_{n}+\sigma \bmod 1$ instead of $x_{n} \oplus \sigma$ has previously been shown by Hickernell [2]. The aim of this work is to analyze a role of $\Phi_{0}(x)=1-|2 x-1|$ in this improvement $O\left(1 / N^{4}\right)$. Generally, we study an influence of u.d.p. maps $\Phi(\mathbf{x})$ on the mean square worst-case error (2). Applying the method of Fourier-Walsh expansion ${ }^{4}$ we express $^{5}$ the mean square worst-case error (2) in the form

$$
\sum_{\substack{\mathbf{k} \in \mathbb{N}_{0}^{s} \\ \mathbf{k} \neq \mathbf{0}}} \widehat{K}_{1}(\mathbf{k}, \mathbf{k})\left|\frac{1}{N} \sum_{n=0}^{N-1} \operatorname{wal}_{\mathbf{k}}\left(\mathbf{x}_{n}\right)\right|^{2},
$$

Supported by APVV Project SK-BG-0019-08.

${ }^{1}$ This is also u.d.p. map.

${ }^{2}$ It called the tent or baker's transformation.

${ }^{3}$ Precisely, for any $\varepsilon>0$ there exists a digital net $x_{0}, \ldots, x_{N-1}$ such that $(1)$ is $O\left(1 / N^{2-\varepsilon}\right)$, and a digital net such that (2) is $O\left(1 / N^{4-\varepsilon}\right)$.

${ }^{4}$ The idea of using Walsh functions stems from Larcher [3].

${ }^{5}$ Partially known formula, see [1; Theorem 4]. 
where $\widehat{K}_{1}(\mathbf{k}, \mathbf{k})$ are Fourier-Walsh coefficients of $K(\Phi(\mathbf{x}), \Phi(\mathbf{y}))$. Here $K(\mathbf{x}, \mathbf{y}), \Phi(\mathbf{x})$ and $\mathbf{x}_{0}, \mathbf{x}_{1}, \ldots$, $\mathbf{x}_{N-1}$ are arbitrary. Thus, in (3) the role of kernel, u.d.p. map and sequence are separated. In Section 3 we apply (3) to the $\Phi(\mathbf{x})=\mathbf{x}, \Phi(\mathbf{x})=\mathbf{b}^{\boldsymbol{\alpha}} \mathbf{x} \bmod 1$ and to a generalized tent function $\Phi(\mathbf{x})$. In Section 4.1, for dimension $s=1$, for kernel $K(x, y)=1-\max (x, y)$ and for the van der Corput sequence $x_{0}, x_{1}, \ldots, x_{N-1}$, we give explicit formulas of the mean square worst-case errors. Theorem 8 shows that the best result is attained for shift $x \oplus \sigma$. In Section $4.2^{6}$ for a Sobolev space with reproducing kernel

$$
K(x, y)=1+\gamma B_{1}(x) B_{1}(y)+\frac{\gamma^{2}}{4} B_{2}(x) B_{2}(y)-\frac{\gamma^{2}}{24} B_{4}(|x-y|)
$$

we see that the rate $O\left(1 / N^{4}\right)$ of mean square worst-case error for the tent u.d.p. map $\Phi_{0}(x)$ is not only given by special properties of $\Phi_{0}(x)$, but it is also a consequence of a selection of exactly $N=2^{\beta}$ initial terms of the van der Corput sequence. Finally, in Theorem 10, for $N=2^{\beta}+1$ we obtain the mean square worst-case error only $O\left(1 / N^{2}\right)$.

Note that in all parts of this paper we carefully compute mean square worst-case errors exactly.

\section{Basic objects}

2.1. Notations. In this paper we use the following notations:

- $s$ is a dimension of the unit cube $[0,1)^{s}$;

- $\mathbf{x}=\left(x_{1}, x_{2}, \ldots, x_{s}\right), \mathbf{y}=\left(y_{1}, y_{2}, \ldots, y_{s}\right)$, and $\boldsymbol{\sigma}=\left(\sigma_{1}, \sigma_{2}, \ldots, \sigma_{s}\right)$ are vectors in $[0,1)^{s}$;

- $\mathbf{k}=\left(k_{1}, k_{2}, \ldots, k_{s}\right)$ is a vector with nonnegative integer coordinates;

- $\mathbf{b}=\left(b_{1}, b_{2}, \ldots, b_{s}\right)$ is a vector with positive integer coordinates; we call it a vector of bases;

- $\mathbf{b}^{\boldsymbol{\alpha}} \mathbf{x} \bmod 1=\left(b_{1}^{\alpha_{1}} x_{1}, \ldots, b_{s}^{\alpha_{s}} x_{s}\right) \bmod 1$, where $\boldsymbol{\alpha}=\left(\alpha_{1}, \ldots, \alpha_{s}\right)$ has nonnegative integers coordinates;

- $\mathbf{b}^{\boldsymbol{\alpha}} \mid \mathbf{k}$ is equivalent to $b_{i}^{\alpha_{i}} \mid k_{i}$ for $i=1,2, \ldots, s$;

- $\mathbf{k} / \mathbf{b}^{\boldsymbol{\alpha}}=\left(k_{1} / b_{1}^{\alpha_{1}}, \ldots, k_{s} / b_{s}^{\alpha_{s}}\right)$;

- $x=x_{0} / b+x_{1} / b^{2}+\cdots$ is a $b$-adic representation of $x \in[0,1)$;

- $\sigma=\sigma_{0} / b+\sigma_{1} / b^{2}+\cdots$;

- $k=k_{0}+k_{1} b+k_{2} b^{2}+\cdots+k_{n} b^{n}$ is a $b$-adic expression of the integer $k, k_{n} \neq 0$;

- $\operatorname{wal}_{k}(x)=e^{(2 \pi i / b)\left(k_{0} x_{0}+k_{1} x_{1}+\cdots+k_{n} x_{n}\right)}$ is the $k$-th Walsh function $\mathrm{wal}_{k}:[0,1] \rightarrow \mathbb{C}$ in the base $b$;

- $\mathrm{wal}_{\mathbf{k}}(\mathbf{x})=\prod_{i=1}^{s} \mathrm{wal}_{k_{i}}\left(x_{i}\right)$;

- $x \oplus \sigma=\left(x_{0}+\sigma_{0}(\bmod b)\right) / b+\left(x_{1}+\sigma_{1}(\bmod b)\right) / b^{2}+\cdots ;$

- $\mathbf{x} \oplus \boldsymbol{\sigma}=\left(x_{1} \oplus \sigma_{1}, x_{2} \oplus \sigma_{2}, \ldots, x_{s} \oplus \sigma_{s}\right)$;

- $\mathbf{x}+\boldsymbol{\sigma} \bmod 1=\left(\left\{x_{1}+\sigma_{1}\right\},\left\{x_{2}+\sigma_{2}\right\}, \ldots,\left\{x_{s}+\sigma_{s}\right\}\right)$, where $\{x\}$ is the fractional part of $x$;

- $\mathbf{x}_{0}, \mathbf{x}_{1}, \ldots, \mathbf{x}_{N-1}$ is an $N$-terms sequence in $[0,1]^{s}$;

- $\mathbb{N}_{0}=\{0,1,2, \ldots\}$;

- if $k=k_{0}+k_{1} b+\cdots+k_{n} b^{n}$ then

$$
-k=-k_{0}-k_{1} b-\cdots-k_{n} b^{n}, \quad \ominus k=k_{0}^{\prime}+k_{1}^{\prime} b+\cdots+k_{n}^{\prime} b^{n},
$$

where $k_{0}+k_{0}^{\prime}=k_{1}+k_{1}^{\prime}=\cdots=k_{n}+k_{n}^{\prime}=0(\bmod b)$.

2.2. Hilbert space with reproducing kernel $\boldsymbol{K}(\mathrm{x}, \mathrm{y})$. The basic properties of Hilbert spaces can be found in monographs [4] and [5]. In this paper we consider the following two Hilbert spaces:

$$
H_{1}=\left\{f:[0,1] \rightarrow \mathbb{R}, f \text { absolutely continuous, } f(1)=0, \int_{0}^{1}\left(f^{\prime}(x)\right)^{2} d x \text { exists }\right\}
$$

\footnotetext{
${ }^{6}$ Using a Fourier-Walsh expansion of the kernel $K\left(\Phi_{0}(x), \Phi_{0}(y)\right)$ in [1; p. 447].
} 
[5; pp. 30-31] with scalar product $f(x) \cdot g(x)=\int_{0}^{1} f^{\prime}(x) g^{\prime}(x) d x$ and reproducing kernel $K(x, y)=$ $1-\max (x, y)$;

$$
H_{2}=\left\{f:[0,1] \rightarrow \mathbb{R}, f \text { absolutely continuous, } \int_{0}^{1}\left(f^{\prime \prime}(x)\right)^{2} d x \text { exists }\right\}
$$

[1] with scalar product

$$
f(x) \cdot g(x)=\int_{0}^{1} f(x) d x \int_{0}^{1} g(x) d x+\frac{1}{\gamma}(f(1)-f(0))(g(1)-g(0))+\frac{1}{\gamma^{2}} \int_{0}^{1} f^{\prime \prime}(x) g^{\prime \prime}(x) d x
$$

and reproducing kernel

$$
K(x, y)=1+\gamma B_{1}(x) B_{1}(y)+\frac{\gamma^{2}}{4} B_{2}(x) B_{2}(y)-\frac{\gamma^{2}}{24} B_{4}(|x-y|),
$$

where $B_{n}$ is the $n$-th Bernoulli polynomial ${ }^{7}$ and $\gamma \in[0,1]$ is the weight. This $H_{2}$ is called the weighted Sobolev space of the order $\alpha=2$.

2.3. Uniform distribution preserving map $\Phi:[\mathbf{0 , 1}]^{s} \rightarrow[\mathbf{0 , 1}]^{s}$. Map $\Phi:[0,1]^{s} \rightarrow[0,1]^{s}$ is called uniform distribution preserving (u.d.p.) map if for every uniformly distributed (u.d.) sequence $\mathbf{x}_{n}, n=1,2, \ldots$, the image $\Phi\left(\mathbf{x}_{n}\right)$ is again u.d. For one-dimensional case basic properties of u.d.p. maps can be found in [6;2.1, (VII)] and [7; 2.5.1].

For example, if $\Phi(x), \Psi(x)$ are u.d.p. transformations and $\alpha$ is a real number, then $\Psi(\Phi(x))$, $1-\Phi(x), \Phi(x)+\alpha \bmod 1$ are also u.d.p. Also, as we mentioned in the Introduction,

$$
\Phi_{0}(x)=1-|2 x-1|, \quad x \in[0,1],
$$

is u.d.p. map called tent map or baker's map, see [2].

Examples of multi-dimensional u.d.p. functions:

(i) $\Phi(\mathbf{x})=\mathbf{x} \oplus \boldsymbol{\sigma}$

(ii) $\Phi(\mathbf{x})=\left(\Phi_{1}\left(x_{1}\right), \ldots, \Phi_{s}\left(x_{s}\right)\right)$, where $\Phi_{n}(x)$ are one-dimensional u.d.p. map;

(iii) $\Phi(\mathbf{x})=\mathbf{b}^{\alpha} \mathbf{x} \bmod 1=\left(b_{1}^{\alpha_{1}} x_{1}, \ldots, b_{s}^{\alpha_{s}} x_{s}\right) \bmod 1$;

(iv) $\Phi(\mathbf{x})=\mathbf{x}+\boldsymbol{\sigma} \bmod 1=\left(x_{1}+\sigma_{1}, \ldots, x_{s}+\sigma_{s}\right) \bmod 1$;

(v) $\Phi(\mathbf{x})=(A \mathbf{x})^{T} \bmod 1$, where $A$ is an $s \times s$ nonsingular integer matrix, cf. Steinerberger [8; Theorem 2];

(vi) $\Phi(\mathbf{x})=\pi(\mathbf{x})$, where $\pi(\mathbf{x})=\left(x_{\pi(1)}, \ldots, x_{\pi(n)}\right)$ is a permutation.

The main criterion of u.d.p. map directly follows from Weyl's limit relation (see [7; pp. 1-62]).

Theorem 1. A map $\Phi(\mathbf{x})$ is u.d.p. if and only if $\Phi(\mathbf{x})$ is Riemann integrable and for every Riemann integrable $f:[0,1]^{s} \rightarrow \mathbb{R}$ we have

$$
\int_{[0,1]^{s}} f(\Phi(\mathbf{x})) d \mathbf{x}=\int_{[0,1]^{s}} f(\mathbf{x}) d \mathbf{x} .
$$

\section{General results}

3.1. The mean square worst-case error and u.d.p. maps $\Psi(\mathrm{x}, \sigma)$. By the Sloan and Woźniakowski [9] formula the square worst-case quasi-Monte Carlo error can be expressed as

$$
\begin{aligned}
\sup _{\substack{f \in H \\
\|f\| \leqslant 1}}\left|\frac{1}{N} \sum_{n=0}^{N-1} f\left(\mathbf{x}_{n}\right)-\int_{[0,1]^{s}} f(\mathbf{x}) d \mathbf{x}\right|^{2} \\
\quad=\int_{[0,1]^{s}} \int_{[0,1]^{s}} K(\mathbf{x}, \mathbf{y}) d \mathbf{x} d \mathbf{y}-\frac{2}{N} \sum_{n=0}^{N-1} \int_{[0,1]^{s}} K\left(\mathbf{x}_{n}, \mathbf{y}\right) d \mathbf{y}+\frac{1}{N^{2}} \sum_{n, m=0}^{N-1} K\left(\mathbf{x}_{m}, \mathbf{x}_{n}\right) .
\end{aligned}
$$

\footnotetext{
${ }^{7}$ Bernoulli polynomials: $B_{1}(x)=x-1 / 2, B_{2}(x)=x^{2}-x+1 / 6, B_{4}(x)=x^{4}-2 x^{3}+x^{2}-1 / 30, \ldots$.
} 
Replacing the sequence $\mathbf{x}_{0}, \ldots, \mathbf{x}_{N-1}$ in (6) by $\Psi\left(\mathbf{x}_{0}, \boldsymbol{\sigma}\right), \ldots, \Psi\left(\mathbf{x}_{N-1}, \boldsymbol{\sigma}\right)$, where $\Psi(\mathbf{x}, \boldsymbol{\sigma})$ is a u.d.p. map with respect to both $\mathbf{x}$ and $\boldsymbol{\sigma}$, and integrating (6) by $\boldsymbol{\sigma}$ the mean square worst-case error can be expressed as

$$
\begin{aligned}
\int_{[0,1]^{s}} & \sup _{\substack{f \in H \\
\|f\| \leqslant 1}}\left|\frac{1}{N} \sum_{n=0}^{N-1} f\left(\Psi\left(\mathbf{x}_{n}, \boldsymbol{\sigma}\right)\right)-\int_{[0,1]^{s}} f(\mathbf{x}) d \mathbf{x}\right|^{2} d \boldsymbol{\sigma} \\
= & \frac{1}{N^{2}} \sum_{n, m=0}^{N-1} \int_{[0,1]^{s}} K\left(\Psi\left(\mathbf{x}_{m}, \boldsymbol{\sigma}\right), \Psi\left(\mathbf{x}_{n}, \boldsymbol{\sigma}\right)\right) d \boldsymbol{\sigma}-\int_{[0,1]^{2 s}} K(\mathbf{x}, \mathbf{y}) d \mathbf{x} d \mathbf{y} .
\end{aligned}
$$

This follows from

$$
\frac{2}{N} \sum_{n=0}^{N-1} \int_{[0,1]^{s}} \int_{[0,1]^{s}} K\left(\Psi\left(\mathbf{x}_{n}, \boldsymbol{\sigma}\right), \mathbf{y}\right) d \mathbf{y} d \boldsymbol{\sigma}=2 \int_{[0,1]^{2 s}} K(\mathbf{x}, \mathbf{y}) d \mathbf{x} d \mathbf{y},
$$

which holds for every u.d.p. map $\Psi(\mathbf{x}, \boldsymbol{\sigma})$ and arbitrary $\mathbf{x}_{0}, \ldots, \mathbf{x}_{N-1}$ in $[0,1)^{s}$, where we apply the u.d.p. characteristic integral equation (5).

3.2. The mean square worst-case error expressed by Fourier-Walsh coefficients. For u.d.p. function of the type $\mathbf{x} \oplus \boldsymbol{\sigma}$ we compute (7) analogously as in [2] using Fourier-Walsh expansion of the kernel $K(\mathbf{x}, \mathbf{y})$, see [5; Theorem 12.4]: ${ }^{8}$

$$
K(\mathbf{x}, \mathbf{y})=\sum_{\mathbf{k}, \mathbf{k}^{\prime} \in \mathbb{N}_{0}^{s}} \widehat{K}\left(\mathbf{k}, \mathbf{k}^{\prime}\right) \overline{\mathrm{wal}_{\mathbf{k}}(\mathbf{x})} \mathrm{wal}_{\mathbf{k}^{\prime}}(\mathbf{y}),
$$

where

$$
\widehat{K}\left(\mathbf{k}, \mathbf{k}^{\prime}\right)=\int_{[0,1]^{2 s}} K(\mathbf{x}, \mathbf{y}) \mathrm{wal}_{\mathbf{k}}(\mathbf{x}) \overline{\mathrm{wal}_{\mathbf{k}^{\prime}}(\mathbf{y})} d \mathbf{x} d \mathbf{y} .
$$

THEOREM 2. For every sequence $\mathbf{x}_{0}, \ldots, \mathbf{x}_{N-1}$ in the unit cube $[0,1)^{s}$ and an arbitrary kernel $K(\mathbf{x}, \mathbf{y})$ with Fourier-Walsh expansion $(9)^{9}$ we have

$$
\int_{[0,1]^{s}} \sup _{\substack{f \in H \\\|f\| \leqslant 1}}\left|\frac{1}{N} \sum_{n=0}^{N-1} f\left(\mathbf{x}_{n} \oplus \boldsymbol{\sigma}\right)-\int_{[0,1]^{s}} f(\mathbf{x}) d \mathbf{x}\right|^{2} d \boldsymbol{\sigma}=\sum_{\substack{\mathbf{k} \in \mathbb{N}_{0}^{s} \\ \mathbf{k} \neq \mathbf{0}}} \widehat{K}(\mathbf{k}, \mathbf{k})\left|\frac{1}{N} \sum_{n=0}^{N-1} \mathrm{wal}_{\mathbf{k}}\left(\mathbf{x}_{n}\right)\right|^{2},
$$

where

$$
\widehat{K}(\mathbf{k}, \mathbf{k})=\int_{[0,1]^{2 s}} K(\mathbf{x}, \mathbf{y}) \mathrm{wal}_{\mathbf{k}}(\mathbf{x}) \overline{\mathrm{wal}_{\mathbf{k}}(\mathbf{y})} d \mathbf{x} d \mathbf{y} .
$$

Proof. Using the following elementary properties of Walsh functions

$$
\begin{gathered}
\text { wal }_{\mathbf{k}}(\mathbf{x} \oplus \boldsymbol{\sigma})=\text { wal }_{\mathbf{k}}(\mathbf{x}) \text { wal }_{\mathbf{k}}(\boldsymbol{\sigma}), \\
\text { wal }_{\mathbf{k} \oplus \mathbf{k}^{\prime}}(\mathbf{x})=\text { wal }_{\mathbf{k}}(\mathbf{x}) \text { wal }_{\mathbf{k}^{\prime}}(\mathbf{x}), \\
\int_{[0,1]^{s}} \overline{\text { wal }_{\mathbf{k}}(\boldsymbol{\sigma})} \text { wal }_{\mathbf{k}^{\prime}}(\boldsymbol{\sigma}) d \boldsymbol{\sigma}= \begin{cases}0 & \text { if } \mathbf{k} \neq \mathbf{k}^{\prime}, \\
1 & \text { if } \mathbf{k}=\mathbf{k}^{\prime},\end{cases}
\end{gathered}
$$

${ }^{8}$ In the literature (see [5; p. 364]) it is preferably used opposite

$$
K(\mathbf{x}, \mathbf{y})=\sum_{\mathbf{k}, \mathbf{k}^{\prime} \in \mathbb{N}_{0}^{S}} \widehat{K}\left(\mathbf{k}, \mathbf{k}^{\prime}\right) \mathrm{wal}_{\mathbf{k}}(\mathbf{x}) \overline{\mathrm{wal}_{\mathbf{k}^{\prime}}(\mathbf{y})}
$$

${ }^{9}$ Assuming that the convergence rate of (9) admits integration by terms. 
we express

$$
\begin{aligned}
& \int_{[0,1]^{s}} K(\mathbf{x} \oplus \boldsymbol{\sigma}, \mathbf{y} \oplus \boldsymbol{\sigma}) d \boldsymbol{\sigma} \\
& =\sum_{\mathbf{k}, \mathbf{k}^{\prime} \in \mathbb{N}_{0}^{s}} \widehat{K}\left(\mathbf{k}, \mathbf{k}^{\prime}\right) \int_{[0,1]^{s}} \overline{\mathrm{wal}_{\mathbf{k}}(\mathbf{x} \oplus \boldsymbol{\sigma})} \mathrm{wal}_{\mathbf{k}^{\prime}}(\mathbf{y} \oplus \boldsymbol{\sigma}) d \boldsymbol{\sigma} \\
& =\sum_{\mathbf{k}, \mathbf{k}^{\prime} \in \mathbb{N}_{0}^{s}} \widehat{K}\left(\mathbf{k}, \mathbf{k}^{\prime}\right) \begin{cases}0 & \text { if } \mathbf{k} \neq \mathbf{k}^{\prime}, \\
\overline{\mathrm{wal}_{\mathbf{k}}(\mathbf{x})} \mathrm{wal}_{\mathbf{k}}(\mathbf{y}) & \text { if } \mathbf{k}=\mathbf{k}^{\prime} .\end{cases}
\end{aligned}
$$

Thus in (7) we have

$$
\int_{[0,1]^{s}} K\left(\mathbf{x}_{m} \oplus \boldsymbol{\sigma}, \mathbf{x}_{n} \oplus \boldsymbol{\sigma}\right) d \boldsymbol{\sigma}=\sum_{\mathbf{k} \in \mathbb{N}_{0}^{s}} \widehat{K}(\mathbf{k}, \mathbf{k}) \overline{\operatorname{wal}_{\mathbf{k}}\left(\mathbf{x}_{m}\right)} \operatorname{wal}_{\mathbf{k}}\left(\mathbf{x}_{n}\right)
$$

and $\widehat{K}(\mathbf{0}, \mathbf{0})=\int_{[0,1]^{2 s}} K(\mathbf{x}, \mathbf{y}) d \mathbf{x} d \mathbf{y}$, and the result (10) follows.

In the following theorem we extend [1; Theorem 4] or [5; Theorem 12.7], which was proved for Sobolev weighted spaces.

TheOREM 3. For every sequence $\mathbf{x}_{0}, \ldots, \mathbf{x}_{N-1}$ in the unit cube $[0,1)^{s}$ and every u.d.p. map $\Phi(\mathbf{x})$ and an arbitrary kernel $K(\mathbf{x}, \mathbf{y})$ with Fourier-Walsh expansion (9) we have

$$
\int_{[0,1]^{s}} \sup _{\substack{f \in H \\\|f\| \leqslant 1}}\left|\frac{1}{N} \sum_{n=0}^{N-1} f\left(\Phi\left(\mathbf{x}_{n} \oplus \boldsymbol{\sigma}\right)\right)-\int_{[0,1]^{s}} f(\mathbf{x}) d \mathbf{x}\right|^{2} d \boldsymbol{\sigma}=\sum_{\substack{\mathbf{k} \in \mathbb{N}_{0}^{s} \\ \mathbf{k} \neq \mathbf{0}}} \widehat{K}_{1}(\mathbf{k}, \mathbf{k})\left|\frac{1}{N} \sum_{n=0}^{N-1} \mathrm{wal}_{\mathbf{k}}\left(\mathbf{x}_{n}\right)\right|^{2},
$$

where

$$
\widehat{K}_{1}(\mathbf{k}, \mathbf{k})=\int_{[0,1]^{2 s}} K(\Phi(\mathbf{x}), \Phi(\mathbf{y})) \mathrm{wal}_{\mathbf{k}}(\mathbf{x}) \overline{\mathrm{wal}_{\mathbf{k}}(\mathbf{y})} d \mathbf{x} d \mathbf{y} .
$$

Proof. We express $K(\Phi(\mathbf{x}), \Phi(\mathbf{y}))$ to the Fourier-Walsh series

$$
K(\Phi(\mathbf{x}), \Phi(\mathbf{y}))=\sum_{\mathbf{k}, \mathbf{k}^{\prime} \in \mathbb{N}_{0}^{s}} \widehat{K}_{1}\left(\mathbf{k}, \mathbf{k}^{\prime}\right) \overline{\mathrm{wal}_{\mathbf{k}}(\mathbf{x})} \mathrm{wal}_{\mathbf{k}^{\prime}}(\mathbf{y}),
$$

where

$$
\widehat{K}_{1}\left(\mathbf{k}, \mathbf{k}^{\prime}\right)=\int_{[0,1]^{2 s}} K(\Phi(\mathbf{x}), \Phi(\mathbf{y})) \mathrm{wal}_{\mathbf{k}}(\mathbf{x}) \overline{\mathrm{wal}_{\mathbf{k}^{\prime}}(\mathbf{y})} d \mathbf{x} d \mathbf{y}
$$

and as in (11) we express ${ }^{10}$

$$
\begin{array}{rl}
\int_{[0,1]^{s}} & K(\Phi(\mathbf{x} \oplus \boldsymbol{\sigma}), \Phi(\mathbf{y} \oplus \boldsymbol{\sigma})) d \boldsymbol{\sigma} \\
\quad= & \sum_{\mathbf{k}, \mathbf{k}^{\prime} \in \mathbb{N}_{0}^{s}} \widehat{K}_{1}\left(\mathbf{k}, \mathbf{k}^{\prime}\right) \int_{[0,1]^{s}} \overline{\operatorname{wal}_{\mathbf{k}}(\mathbf{x} \oplus \boldsymbol{\sigma})} \operatorname{wal}_{\mathbf{k}^{\prime}}(\mathbf{y} \oplus \boldsymbol{\sigma}) d \boldsymbol{\sigma}=\sum_{\mathbf{k} \in \mathbb{N}_{0}^{s}} \widehat{K}_{1}(\mathbf{k}, \mathbf{k}) \overline{\mathrm{wal}_{\mathbf{k}}(\mathbf{x})} \mathrm{wal}_{\mathbf{k}}(\mathbf{y})
\end{array}
$$

and then by (7) now we find (12).

${ }^{10}$ In [1] or [5; Theorem 12.7] the authors use a new kernel

$$
K^{*}(\mathbf{x}, \mathbf{y})=\int_{[0,1]^{s}} K(\Phi(\mathbf{x} \oplus \boldsymbol{\sigma}), \Phi(\mathbf{y} \oplus \boldsymbol{\sigma})) d \boldsymbol{\sigma} .
$$

We have $\widehat{K^{*}}(\mathbf{k}, \mathbf{k})=\widehat{K}_{1}(\mathbf{k}, \mathbf{k})$ and thus Theorem 3 is a consequence of Theorem 2 . 
3.3. U.d.p. map $\Phi(\mathbf{x} \oplus \boldsymbol{\sigma})$, where $\Phi(\mathbf{x})=\mathbf{b}^{\alpha} \mathbf{x} \bmod 1$. Here $\mathbf{x} \oplus \boldsymbol{\sigma}$ and wal $_{\mathbf{k}}(\mathbf{x})$ are in the same base $\mathbf{b}$.

ThEOREM 4. For $\Phi(\mathbf{x})=\mathbf{b}^{\boldsymbol{\alpha}} \mathbf{x} \bmod 1$ and for a kernel $K(\mathbf{x}, \mathbf{y})$ of the type

$$
K(\mathbf{x}, \mathbf{y})=\prod_{j=1}^{s} K_{j}\left(x_{j}, y_{j}\right)
$$

and for an arbitrary sequence $\mathbf{x}_{0}, \mathbf{x}_{1}, \ldots, \mathbf{x}_{N-1}$ in $[0,1)^{s}$ we have

$$
\begin{aligned}
\int_{[0,1]^{s}} & \sup _{\substack{f \in H \\
\|f\| \leqslant 1}}\left|\frac{1}{N} \sum_{n=0}^{N-1} f\left(\Phi\left(\mathbf{x}_{n} \oplus \boldsymbol{\sigma}\right)\right)-\int_{[0,1]^{s}} f(\mathbf{x}) d \mathbf{x}\right|^{2} d \boldsymbol{\sigma} \\
= & \sum_{\substack{\mathbf{k} \in \mathbb{N}_{0}^{s} \\
\mathbf{k} \neq \mathbf{0}, \mathbf{b}^{\boldsymbol{\alpha}} \mid \mathbf{k}}} \widehat{K}\left(\frac{\mathbf{k}}{\mathbf{b}^{\boldsymbol{\alpha}}}, \frac{\mathbf{k}}{\mathbf{b}^{\boldsymbol{\alpha}}}\right)\left|\frac{1}{N} \sum_{n=0}^{N-1} \mathrm{wal}_{\mathbf{k}}\left(\mathbf{x}_{n}\right)\right|^{2} \\
= & \sum_{\substack{\mathbf{k} \in \mathbb{N}_{0}^{s} \\
\mathbf{k} \neq \mathbf{0}}} \widehat{K}(\mathbf{k}, \mathbf{k})\left|\frac{1}{N} \sum_{n=0}^{N-1} \operatorname{wal}_{\mathbf{k}}\left(\Phi\left(\mathbf{x}_{n}\right)\right)\right|^{2}
\end{aligned}
$$

where

$$
\widehat{K}(\mathbf{k}, \mathbf{k})=\int_{[0,1]^{2 s}} K(\mathbf{x}, \mathbf{y}) \mathrm{wal}_{\mathbf{k}}(\mathbf{x}) \overline{\mathrm{wal}_{\mathbf{k}}(\mathbf{y})} d \mathbf{x} d \mathbf{y} .
$$

Proof. We use Theorem 3. For this we need to compute Fourier-Walsh series

$$
K(\Phi(\mathbf{x}), \Phi(\mathbf{y}))=\sum_{\mathbf{k}, \mathbf{k}^{\prime} \in \mathbb{N}_{0}^{s}} \widehat{K}_{1}\left(\mathbf{k}, \mathbf{k}^{\prime}\right) \overline{\mathrm{wal}_{\mathbf{k}}(\mathbf{x})} \mathrm{wal}_{\mathbf{k}^{\prime}}(\mathbf{y}),
$$

what follows from the one-dimensional Fourier-Walsh series for the kernel

$$
K(\Phi(x), \Phi(y))=\sum_{k, k^{\prime} \in \mathbb{N}_{0}} \widehat{K}_{1}\left(k, k^{\prime}\right) \overline{\operatorname{wal}_{k}(x)} \operatorname{wal}_{k^{\prime}}(y)
$$

where u.d.p. $\Phi(x)=b^{i} x \bmod 1$. For coefficient $\widehat{K}_{1}\left(k, k^{\prime}\right)$ we have

$$
\begin{aligned}
\widehat{K}_{1}\left(k, k^{\prime}\right) & =\int_{0}^{1} \int_{0}^{1} K(\Phi(x), \Phi(y)) \mathrm{wal}_{k}(x) \overline{\mathrm{wal}_{k^{\prime}}(y)} d x d y \\
& = \begin{cases}K\left(\frac{k}{b^{i}}, \frac{k^{\prime}}{b^{i}}\right)=\int_{0}^{1} \int_{0}^{1} K(x, y) \mathrm{wal}_{k / b^{i}}(x) \overline{\mathrm{wal}_{k^{\prime} / b^{i}}(y)} d x d y & \text { if } b^{i} \mid k \text { and } b^{i} \mid k^{\prime}, \\
0 & \text { if } b^{i} \nmid k \text { or } b^{i} \nmid k^{\prime} .\end{cases}
\end{aligned}
$$

Proof of (15). We have

$$
\begin{aligned}
& \int_{0}^{1} \int_{0}^{1} K(\Phi(x), \Phi(y)) \operatorname{wal}_{k}(x) \overline{\operatorname{wal}_{k^{\prime}}(y)} d x d y \\
& \quad=\sum_{\alpha=0}^{b^{i}-1} \sum_{\beta=0}^{b^{i}-1} \int_{\alpha / b^{i}}^{(\alpha+1) / b^{i}} \int_{\beta / b^{i}}^{(\beta+1) / b^{i}} K\left(b^{i} x-\alpha, b^{i} y-\beta\right) \operatorname{wal}_{k}(x) \overline{\operatorname{wal}_{k^{\prime}}(y)} d x d y
\end{aligned}
$$


and by substitutions $b^{i} x-\alpha=u, b^{i} x-\beta=v$ we find

$$
\sum_{\alpha=0}^{b^{i}-1} \sum_{\beta=0}^{b^{i}-1} \int_{0}^{1} \int_{0}^{1} K(u, v) \frac{1}{b^{2 i}} \operatorname{wal}_{k}\left(\frac{u+\alpha}{b^{i}}\right) \overline{\operatorname{wal}_{k^{\prime}}\left(\frac{v+\beta}{b^{i}}\right)} d u d v .
$$

For

$$
\begin{gathered}
\frac{\alpha}{b^{i}}=\frac{\alpha_{0}}{b}+\frac{\alpha_{1}}{b^{2}}+\cdots+\frac{\alpha_{i-1}}{b^{i}} \\
u=\frac{u_{0}}{b}+\frac{u_{1}}{b^{2}}+\cdots, \quad k=k_{0}+k_{1} b+\cdots+k_{n-1} b^{n-1}
\end{gathered}
$$

we have

$$
\frac{u+\alpha}{b^{i}}=\frac{\alpha_{0}}{b}+\frac{\alpha_{1}}{b^{2}}+\cdots+\frac{\alpha_{i-1}}{b^{i}}+\frac{u_{0}}{b^{i+1}}+\frac{u_{1}}{b^{i+2}}+\cdots,
$$

which for Walsh function $\operatorname{wal}_{k}(x)$ in base $b$ gives

$$
\begin{aligned}
\operatorname{wal}_{k}\left(\frac{u+\alpha}{b^{i}}\right) & =e^{(2 \pi i / b)\left(k_{0} \alpha_{0}+\cdots+k_{i-1} \alpha_{i-1}+k_{i} u_{0}+\cdots+k_{n-1} u_{n-1-i}\right)} \\
& =e^{(2 \pi i / b)\left(k_{0} \alpha_{0}+\cdots+k_{i-1} \alpha_{i-1}\right)} \operatorname{wal}_{\left[k / b^{i}\right]}(u),
\end{aligned}
$$

where $\left[k / b^{i}\right]$ is the integer part of $k / b^{i}$. Thus

$$
\begin{gathered}
\sum_{\alpha=0}^{b^{i}-1} \sum_{\beta=0}^{b^{i}-1} \int_{0}^{1} \int_{0}^{1} K(u, v) \frac{1}{b^{2 i}} \operatorname{wal}_{k}\left(\frac{u+\alpha}{b^{i}}\right) \overline{\operatorname{wal}_{k^{\prime}}\left(\frac{v+\beta}{b^{i}}\right)} d u d v \\
=\frac{1}{b^{2 i}} \sum_{\alpha=0}^{b^{i}-1} e^{(2 \pi i / b)\left(k_{0} \alpha_{0}+\cdots+k_{i-1} \alpha_{i-1}\right)} \sum_{\beta=0}^{b^{i}-1} e^{-(2 \pi i / b)\left(k_{0}^{\prime} \beta_{0}+\cdots+k_{i-1}^{\prime} \beta_{i-1}\right)} \\
\times \int_{0}^{1} \int_{0}^{1} K(u, v) \operatorname{wal}_{\left[k / b^{i}\right]}(u) \overline{\operatorname{wal}_{\left[k^{\prime} / b^{i}\right]}(v)} d u d v
\end{gathered}
$$

Since

$$
\sum_{\alpha=0}^{b^{i}-1} e^{(2 \pi i / b)\left(k_{0} \alpha_{0}+\cdots+k_{i-1} \alpha_{i-1}\right)}=\left(\sum_{\alpha_{0}=0}^{b-1} e^{(2 \pi i / b) k_{0} \alpha_{0}}\right) \cdots\left(\sum_{\alpha_{i-1}=0}^{b-1} e^{(2 \pi i / b) k_{i-1} \alpha_{i-1}}\right)
$$

and the right hand side of (16) is non-zero if and only if $b\left|k_{0}, \ldots, b\right| k_{i-1}$, which implies $k_{0}=\cdots=k_{i-1}=0$, and this is equivalent $b^{i} \mid k$, which gives (15).

Now, using (15), assumption $K(\mathbf{x}, \mathbf{y})=\prod_{j=1}^{s} K_{j}\left(x_{j}, y_{j}\right)$ and that u.d.p. map has the form $\Phi(\mathbf{x})=\left(\Phi_{1}\left(x_{1}\right), \ldots, \Phi_{s}\left(x_{s}\right)\right)$, then for Fourier-Walsh coefficients $\widehat{K}_{1}\left(\mathbf{k}, \mathbf{k}^{\prime}\right)$ of $K(\Phi(\mathbf{x}), \Phi(\mathbf{y}))$ we have

$$
\begin{aligned}
\widehat{K}_{1}\left(\mathbf{k}, \mathbf{k}^{\prime}\right) & =\int_{[0,1]^{2 s}} K(\Phi(\mathbf{x}), \Phi(\mathbf{y})) \mathrm{wal}_{\mathbf{k}}(\mathbf{x}) \overline{\mathrm{wal}_{\mathbf{k}^{\prime}}(\mathbf{y})} d \mathbf{x} d \mathbf{y} \\
& =\prod_{j=1}^{s} \widehat{K}_{1, j}\left(x_{j}, y_{j}\right)=\prod_{j=1}^{s} \widehat{K}_{j}\left(\frac{x_{j}}{b_{j}^{\alpha_{j}}}, \frac{y_{j}}{b_{j}^{\alpha_{j}}}\right)=\widehat{K}\left(\frac{\mathbf{k}}{\mathbf{b}^{\boldsymbol{\alpha}}}, \frac{\mathbf{k}^{\prime}}{\mathbf{b}^{\boldsymbol{\alpha}}}\right)
\end{aligned}
$$

if $\mathbf{b}^{\boldsymbol{\alpha}} \mid \mathbf{k}$ and $\mathbf{b}^{\boldsymbol{\alpha}} \mid \mathbf{k}^{\prime}$. Others $\widehat{K}_{1}\left(\mathbf{k}, \mathbf{k}^{\prime}\right)=0$. This implies

$$
K(\Phi(\mathbf{x}), \Phi(\mathbf{y}))=\sum_{\substack{\mathbf{k}, \mathbf{k}^{\prime} \in \mathbb{N}_{0}^{s} \\ \mathbf{b}^{\alpha}\left|\mathbf{k}, \mathbf{b}^{\alpha}\right| \mathbf{k}^{\prime}}} \widehat{K}\left(\frac{\mathbf{k}}{\mathbf{b}^{\boldsymbol{\alpha}}}, \frac{\mathbf{k}^{\prime}}{\mathbf{b}^{\boldsymbol{\alpha}}}\right) \overline{\mathrm{wal}_{\mathbf{k}}(\mathbf{x})} \operatorname{wal}_{\mathbf{k}}(\mathbf{y})
$$

Then by (12) from (17) follows (13). 
The expression (14) directly follows from that $\mathbf{b}^{\boldsymbol{\alpha}} \mid \mathbf{k}$ implies

$$
\mathrm{wal}_{\mathbf{k}}(\mathbf{x})=\mathrm{wal}_{\mathbf{k} / \mathbf{b}^{\alpha}}\left(\mathbf{b}^{\alpha} \mathbf{x} \bmod 1\right) .
$$

Proof of (18). Assume that for $s=1$

$$
k=k_{0}+k_{1} b+\cdots+k_{n} b^{n}, \quad x=\frac{x_{0}}{b}+\frac{x_{1}}{b^{2}}+\cdots,
$$

then $\operatorname{wal}_{k}(x)=e^{(2 \pi i / b)\left(k_{0} x_{0}+k_{1} x_{1}+\cdots+k_{n} x_{n}\right)}, b^{i} \mid k$ equal to $k_{0}=k_{1}=\cdots=k_{i-1}=0$,

$$
b^{i} x=x_{0} b^{i-1}+x_{1} b^{i-2}+\cdots+x_{i-1}+\frac{x_{i}}{b}+\cdots
$$

then

$$
e^{(2 \pi i / b)\left(k_{i} x_{i}+k_{i+1} x_{i+1}+\ldots\right)}=\operatorname{wal}_{k / b^{i}}\left(\left\{b^{i} x\right\}\right) .
$$

This completes the proof of Theorem 4 .

EXAmple. Let $\mathbf{x}_{0}, \ldots, \mathbf{x}_{N-1}$ be a sequence in $[0,1)^{s}$ such that every coordinate $x_{i, n}$ of $\mathbf{x}_{n}$ has the form

$$
x_{i, n}=\frac{a_{i, n}}{b_{i}^{\alpha_{i}}}, \quad 0 \leqslant a_{i, n}<b_{i}^{\alpha_{i}}, \quad a_{i, n} \in \mathbb{N}_{0} .
$$

Then (13) has the form

$$
\begin{gathered}
\int_{[0,1]^{s}} \sup _{\substack{f \in H \\
\|f\| \leqslant 1}}\left|\frac{1}{N} \sum_{n=0}^{N-1} f\left(\Phi\left(\mathbf{x}_{n} \oplus \boldsymbol{\sigma}\right)\right)-\int_{[0,1]^{s}} f(\mathbf{x}) d \mathbf{x}\right|^{2} d \boldsymbol{\sigma} \\
=\int_{[0,1]^{s}} K(\mathbf{x}, \mathbf{x}) d \mathbf{x}-\int_{[0,1]^{2 s}} K(\mathbf{x}, \mathbf{y}) d \mathbf{x} d \mathbf{y} .
\end{gathered}
$$

Proof. For an arbitrary term $\mathbf{x}_{n}$ of the sequence (19) we have $\left(\mathbf{b}^{\alpha} \mathbf{x}_{n} \bmod 1\right)=\mathbf{0}$ and thus for an arbitrary $\mathbf{k}$ we have wal $_{\mathbf{k}}\left(\mathbf{b}^{\boldsymbol{\alpha}} \mathbf{x}_{n} \bmod 1\right)=1$. Thus, for the sum (13) we have

$$
\begin{aligned}
\sum_{\substack{\mathbf{k} \in \mathbb{N}_{0}^{s} \\
\mathbf{k} \neq \mathbf{0}}} \widehat{K}(\mathbf{k}, \mathbf{k}) & =\sum_{\substack{\mathbf{k} \in \mathbb{N}_{0}^{s} \\
\mathbf{k} \neq \mathbf{0}}} \widehat{K}(\mathbf{k}, \mathbf{k}) \overline{\mathrm{wal}_{\mathbf{k}}(\mathbf{0})} \mathrm{wal}_{\mathbf{k}}(\mathbf{0}) \\
& =\int_{[0,1]^{s}} K(\mathbf{0} \oplus \boldsymbol{\sigma}, \mathbf{0} \oplus \boldsymbol{\sigma}) d \boldsymbol{\sigma}-\widehat{K}(\mathbf{0}, \mathbf{0})=\int_{[0,1]^{s}} K(\mathbf{x}, \mathbf{x}) d \mathbf{x}-\widehat{K}(\mathbf{0}, \mathbf{0})
\end{aligned}
$$

and $\widehat{K}(\mathbf{0}, \mathbf{0})=\int_{[0,1]^{2 s}} K(\mathbf{x}, \mathbf{y}) d \mathbf{x} d \mathbf{y}$.

REMARK 1. Hickernell [10] notes that the mean square worst-case error with respect to all sequences $\mathbf{x}_{0}, \ldots, \mathbf{x}_{N-1}$ in $[0,1)^{s}$ becomes

$$
\begin{gathered}
\int_{[0,1]^{s N}}\left(\sup _{\substack{f \in H \\
\|f\| \leqslant 1}}\left|\frac{1}{N} \sum_{n=0}^{N-1} f\left(\mathbf{x}_{n}\right)-\int_{[0,1]^{s}} f(\mathbf{x}) d \mathbf{x}\right|^{2}\right) d \mathbf{x}_{0} \ldots d \mathbf{x}_{N-1} \\
=\frac{1}{N}\left(\int_{[0,1]^{s}} K(\mathbf{x}, \mathbf{x}) d \mathbf{x}-\int_{[0,1]^{2 s}} K(\mathbf{x}, \mathbf{y}) d \mathbf{x} d \mathbf{y}\right) .
\end{gathered}
$$

Note that the error (20) of the sequence (19) is much worse than the mean square worst-case error in $(22)$. 
3.4. U.d.p. map $\Phi(\mathrm{x} \oplus \boldsymbol{\sigma})$, where $\Phi(\mathrm{x})$ is the tent map. The simple tent map $\Phi_{0}(x)$ in (4) can be extended to the map $\Phi(x)$ with the graph in Fig. 1 and defined by following: Putting

$$
I_{i}=\left[\frac{i-1}{b}, \frac{i}{b}\right), \quad i=1,2, \ldots, b,
$$

we define

$$
\Phi(x)= \begin{cases}\Phi_{i}(x)=b x-(i-1) & \text { if } x \in I_{i}, 2 \mid i \\ \Psi_{i}(x)=-b x+i & \text { if } x \in I_{i}, 2 \nmid i\end{cases}
$$

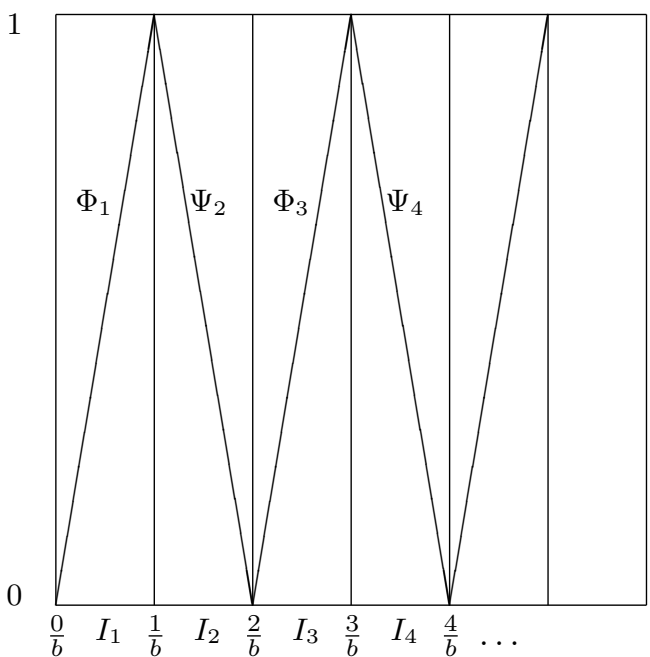

Fig. 1. Graph of $\Phi(x)$ tent function

Remind that if $k=k_{0}+k_{1} b+\cdots+k_{n} b^{n}$, then

$$
-k=-k_{0}-k_{1} b-\cdots-k_{n} b^{n}, \quad \ominus k=k_{0}^{\prime}+k_{1}^{\prime} b+\cdots+k_{n}^{\prime} b^{n},
$$

where $k_{0}+k_{0}^{\prime}=k_{1}+k_{1}^{\prime}=\cdots=k_{n}+k_{n}^{\prime}=0(\bmod b)$. If

$$
x=\frac{x_{0}}{b}+\frac{x_{1}}{b^{2}}+\cdots+\frac{x_{n}}{b^{n+1}}+\cdots,
$$

then

$$
\text { wal }_{-k}(x)=e^{(2 \pi i / b)\left(-k_{0} x_{0}-k_{1} x_{1}-\cdots-k_{n} x_{n}\right)}=\operatorname{wal}_{\ominus k}(x)=\overline{\operatorname{wal}_{k}(x)}
$$

and in the following we use, e.g., $\widehat{K}(k,-k)$ instead of $\widehat{K}(k, \ominus k)$.

THEOREM 5. Let $K(x, y)$ be an arbitrary continuous kernel with a Fourier-Walsh series

$$
K(x, y)=\sum_{k, k^{\prime} \in \mathbb{N}_{0}} \widehat{K}\left(k, k^{\prime}\right) \overline{\mathrm{wal}_{k}(x)} \mathrm{wal}_{k^{\prime}}(y)
$$

in base $b, 2 \mid b$, and let $\Phi(x)$ be the tent function (23). Then for every sequence $x_{0}, \ldots, x_{N-1}$ in the unit interval $[0,1)$ we have

$$
\int_{0}^{1} \sup _{\substack{f \in H \\\|f\| \leqslant 1}}\left|\frac{1}{N} \sum_{n=0}^{N-1} f\left(\Phi\left(x_{n} \oplus \sigma\right)\right)-\int_{0}^{1} f(x) d x\right|^{2} d \sigma=\sum_{k=1}^{\infty} \widehat{K}_{1}(k, k)\left|\frac{1}{N} \sum_{n=0}^{N-1} \operatorname{wal}_{k}\left(x_{n}\right)\right|^{2}
$$


where $^{11}$

$$
\begin{gathered}
\widehat{K}_{1}(k, k)=\frac{1}{4}\left(\widehat{K}\left(\left[\frac{k}{b}\right],\left[\frac{k}{b}\right]\right)+\widehat{K}\left(-\left[\frac{k}{b}\right],-\left[\frac{k}{b}\right]\right)+\widehat{K}\left(\left[\frac{k}{b}\right],-\left[\frac{k}{b}\right]\right) e^{(2 \pi i / b)\left(k_{0}+k_{1}+\cdots+k_{n}\right)}\right. \\
\left.+\widehat{K}\left(-\left[\frac{k}{b}\right],\left[\frac{k}{b}\right]\right) e^{-(2 \pi i / b)\left(k_{0}+k_{1}+\cdots+k_{n}\right)}\right)
\end{gathered}
$$

for $(b / 2) \mid k$ and $\widehat{K}_{1}(k, k)=0$ otherwise. Here $k=k_{0}+k_{1} b+\cdots+k_{n} b^{n}, k_{n} \neq 0$ and $x \oplus \sigma$ and $\Phi(x)$ are in the same even base $b$.

Proof. We start with Fourier-Walsh expansion

$$
K(\Phi(x), \Phi(y))=\sum_{k, k^{\prime} \in \mathbb{N}_{0}} \widehat{K}_{1}\left(k, k^{\prime}\right) \overline{\operatorname{wal}_{k}(x)} \operatorname{wal}_{k^{\prime}}(y),
$$

where

$$
\widehat{K}_{1}\left(k, k^{\prime}\right)=\int_{0}^{1} \int_{0}^{1} K(\Phi(x), \Phi(y)) \operatorname{wal}_{k}(x) \overline{\operatorname{wal}_{k^{\prime}}(y)} d x d y .
$$

Then by the graph in Fig. 1 of the tent function $\Phi(x)$ we express

$$
\begin{aligned}
\int_{0}^{1} \int_{0}^{1} K(\Phi(x), \Phi(y)) \mathrm{wal}_{k}(x) \overline{\operatorname{wal}_{k^{\prime}}(y)} d x d y \\
\quad=\sum_{\substack{i, j=1 \\
i, j \text { odd }}}^{b} \iint_{I_{i} \times I_{j}}+\sum_{\substack{i, j=1 \\
i, j \text { even }}}^{b} \iint_{I_{i} \times I_{j}}+\sum_{\substack{i, j=1 \\
i \text { odd }, j \text { even }}}^{b} \iint_{I_{i} \times I_{j}}+\sum_{\substack{i, j=1 \\
i \text { even, } j \text { odd }}}^{b} \iint_{I_{i} \times I_{j}} .
\end{aligned}
$$

Thus, we have distinguish four cases, namely I-IV.

I) If $i, j$ both odd, the first integral in (26) is of the form

$$
\begin{aligned}
\iint_{I_{i} \times I_{j}} K\left(\Phi_{i}(x), \Phi_{j}(y)\right) \mathrm{wal}_{k}(x) \overline{\mathrm{wal}_{k^{\prime}}(y)} d x d y \\
=\int_{0}^{1} \int_{0}^{1} K(x, y) \operatorname{wal}_{k}\left(\Phi_{i}^{-1}(x)\right) \overline{\operatorname{wal}_{k^{\prime}}\left(\Phi_{j}^{-1}(y)\right)}\left|d \Phi_{i}^{-1}(x) d \Phi_{j}^{-1}(y)\right| \\
=\int_{0}^{1} \int_{0}^{1} K(x, y) e^{(2 \pi i / b) k_{0}(i-1)} \operatorname{wal}_{[k / b]}(x) e^{(2 \pi i / b) k_{0}^{\prime}(j-1)} \overline{\operatorname{wal}_{\left[k^{\prime} / b\right]}(x)} \frac{d x}{b} \frac{d y}{b} .
\end{aligned}
$$

In (27), for $k=k_{0}+k_{1} b+k_{2} b^{2}+\cdots+k_{n} b^{n}$, we use

$$
\operatorname{wal}_{k}\left(\Phi_{i}^{-1}(x)\right)=e^{(2 \pi i / b) k_{0}(i-1)} \operatorname{wal}_{[k / b]}(x) .
$$

Proof of (28). By Fig. 2, $\operatorname{wal}_{k}\left(\Phi_{i}^{-1}(x)\right)=\operatorname{wal}_{k}((i-1) / b+x / b)$ and from

$$
\begin{gathered}
x=\frac{x_{0}}{b}+\frac{x_{1}}{b^{2}}+\frac{x_{2}}{b^{3}}+\cdots, \quad \frac{x}{b}=\frac{x_{0}}{b^{2}}+\frac{x_{1}}{b^{3}}+\frac{x_{2}}{b^{4}}+\cdots, \\
\frac{i-1}{b}+\frac{x}{b}=\frac{i-1}{b}+\frac{x_{0}}{b^{2}}+\frac{x_{1}}{b^{3}}+\frac{x_{2}}{b^{4}}+\cdots,
\end{gathered}
$$

${ }^{11}$ Alternately

$$
\widehat{K}_{1}(k, k)=\frac{1}{2}\left(\widehat{K}\left(\left[\frac{k}{b}\right],\left[\frac{k}{b}\right]\right)+\operatorname{Re}\left(\widehat{K}\left(\left[\frac{k}{b}\right],-\left[\frac{k}{b}\right]\right) e^{(2 \pi i / b)\left(k_{0}+k_{1}+\cdots+k_{n}\right)}\right)\right) .
$$




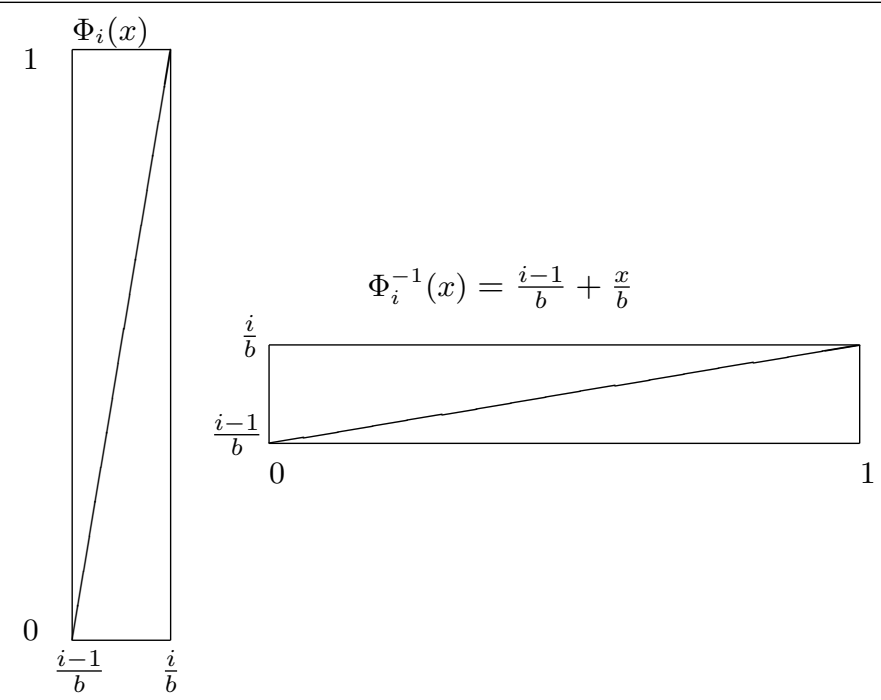

Fig. 2. $\Phi_{i}(x)$ and inverse function of $\Phi_{i}(x)$

and by the definition of Walsh functions we have

$$
\begin{aligned}
\operatorname{wal}_{k}\left(\frac{i-1}{b}+\frac{x}{b}\right) & =e^{(2 \pi i / b)\left(k_{0}(i-1)+k_{1} x_{0}+k_{2} x_{1}+k_{3} x_{2}+\cdots\right)} \\
& =e^{(2 \pi i / b) k_{0}(i-1)} e^{(2 \pi i / b)\left(k_{1} x_{0}+k_{2} x_{1}+\cdots+k_{n} x_{n-1}\right)}=e^{(2 \pi i / b) k_{0}(i-1)} \operatorname{wal}_{[k / b]}(x) .
\end{aligned}
$$

Summing (27) over $\sum_{\substack{i, j=1 \\ i, j \text { odd }}}^{b}$ and from

$$
\sum_{\substack{i=1 \\ i \text { odd }}}^{b} e^{(2 \pi i / b) k_{0}(i-1)}= \begin{cases}\frac{b}{2} & \text { if } \frac{b}{2} \mid k_{0} \quad\left(\Leftrightarrow \frac{b}{2} \mid k\right) \\ 0 & \text { otherwise }\end{cases}
$$

we find

$$
\begin{aligned}
& \sum_{\substack{i, j=1 \\
i, j \text { odd }}}^{b} \iint_{I_{i} \times I_{j}} K\left(\Phi_{i}(x), \Phi_{j}(y)\right) \operatorname{wal}_{k}(x) \overline{\operatorname{wal}_{k^{\prime}}(y)} d x d y \\
& = \begin{cases}\frac{1}{4} \widehat{K}\left(\left[\frac{k}{b}\right],\left[\frac{k^{\prime}}{b}\right]\right) & \text { if } \frac{b}{2}\left|k, \frac{b}{2}\right| k^{\prime}, \\
0 & \text { otherwise. }\end{cases}
\end{aligned}
$$

This gives the first term in the parenthesis (25).

II) If $i, j$ both even, then

$$
\begin{aligned}
& \iint_{I_{i} \times I_{j}} K\left(\Psi_{i}(x), \Psi_{j}(y)\right) \operatorname{wal}_{k}(x) \overline{\operatorname{wal}_{k^{\prime}}(y)} d x d y \\
& =\int_{0}^{1} \int_{0}^{1} K(x, y) \operatorname{wal}_{k}\left(\Psi_{i}^{-1}(x) \overline{\operatorname{wal}_{k^{\prime}}\left(\Psi_{j}^{-1}(y)\right)}\left|d \Psi_{i}^{-1}(x) d \Psi_{j}^{-1}(y)\right|\right. \\
& =\int_{0}^{1} \int_{0}^{1} K(x, y) e^{(2 \pi i / b) k_{0}(i-1)} e^{-(2 \pi i / b)\left(k_{1}+k_{2}+\cdots+k_{n}\right)} \overline{\operatorname{wal}_{[k / b]}(x)} \\
& \quad \times e^{-(2 \pi i / b) k_{0}^{\prime}(j-1)} e^{(2 \pi i / b)\left(k_{1}^{\prime}+k_{2}^{\prime}+\cdots+k_{n^{\prime}}^{\prime}\right)} \operatorname{wal}_{\left[k^{\prime} / b\right]}(y)\left|\frac{d x}{(-b)} \frac{d y}{(-b)}\right|
\end{aligned}
$$


In (30) we use

$$
\operatorname{wal}_{k}\left(\Psi_{i}^{-1}(x)\right)=e^{(2 \pi i / b) k_{0}(i-1)} e^{-(2 \pi i / b)\left(k_{1}+k_{2}+\cdots+k_{n}\right)} \overline{\operatorname{wal}_{[k / b]}(x)} .
$$

Proof of (31). By Fig. 3, $\operatorname{wal}_{k}\left(\Psi_{i}^{-1}(x)\right)=\operatorname{wal}_{k}(i / b-x / b)$, and from

$$
\begin{aligned}
x=\frac{x_{0}}{b}+\frac{x_{1}}{b^{2}}+\frac{x_{2}}{b^{3}}+\cdots \\
\frac{i}{b}-\frac{x}{b}=\frac{i}{b}-\frac{x_{0}}{b^{2}}-\frac{x_{1}}{b^{3}}-\frac{x_{2}}{b^{4}}-\cdots \\
=\frac{i-1}{b}+\left(\frac{1}{b}-\frac{x_{0}}{b}-\frac{x_{1}}{b^{2}}-\frac{x_{2}}{b^{3}}-\cdots\right) \\
=\frac{i-1}{b}+\left(\frac{b-1}{b^{2}}+\frac{b-1}{b^{3}}+\frac{b-1}{b^{4}}+\cdots-\frac{x_{0}}{b}-\frac{x_{1}}{b^{2}}-\frac{x_{2}}{b^{3}}-\cdots\right) \\
=\frac{i-1}{b}+\left(\frac{b-1-x_{0}}{b^{2}}+\frac{b-1-x_{1}}{b^{3}}+\frac{b-1-x_{2}}{b^{4}}+\cdots\right),
\end{aligned}
$$

and by the definition of Walsh functions we have

$$
\begin{aligned}
\operatorname{wal}_{k}\left(\frac{i}{b}-\frac{x}{b}\right) & =e^{(2 \pi i / b)\left(k_{0}(i-1)+k_{1}\left(b-1-x_{0}\right)+k_{2}\left(b-1-x_{1}\right)+\cdots\right)} \\
& =e^{(2 \pi i / b) k_{0}(i-1)} e^{-(2 \pi i / b)\left(k_{1}+k_{2}+\cdots+k_{n}\right)} \overline{\operatorname{wal}_{[k / b]}(x)} .
\end{aligned}
$$

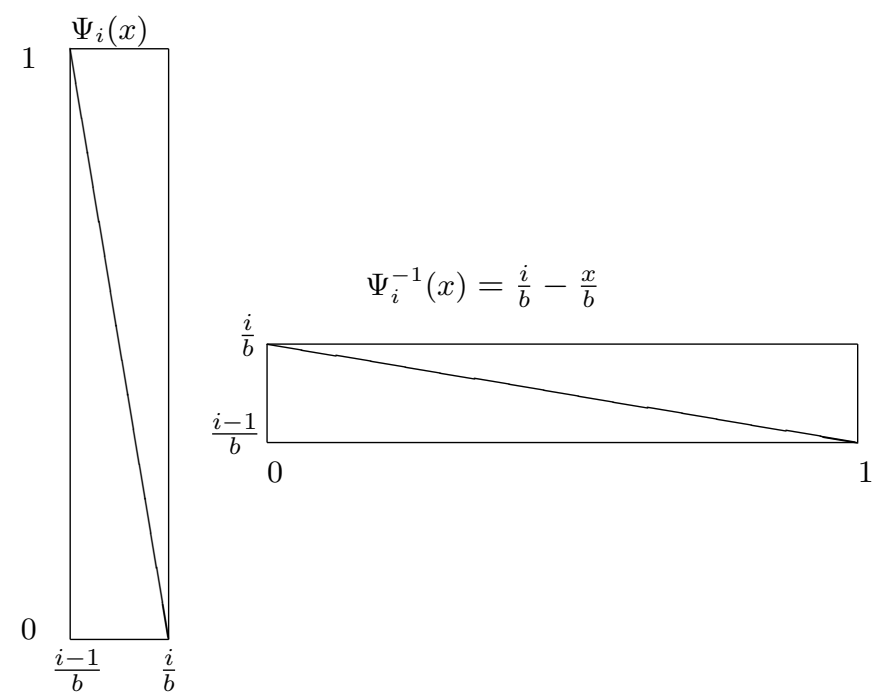

Fig. 3. $\Psi_{i}(x)$ and inverse function of $\Psi_{i}(x)$

Submitting in (30) the sum

$$
\sum_{\substack{i=1 \\ i \text { even }}}^{b} e^{(2 \pi i / b) k_{0}(i-1)}= \begin{cases}e^{-(2 \pi i / b) k_{0}} \frac{b}{2} & \text { if } \frac{b}{2} \mid k_{0} \quad\left(\Leftrightarrow \frac{b}{2} \mid k\right), \\ 0 & \text { otherwise, }\end{cases}
$$

and

$$
\int_{0}^{1} \int_{0}^{1} K(x, y) \overline{\operatorname{wal}_{[k / b]}(x)} \operatorname{wal}_{\left[k^{\prime} / b\right]}(y) d x d y=\widehat{K}(i, j),
$$


where $i \oplus[k / b]=0, j \oplus\left[k^{\prime} / b\right]=0$, we find

$$
\begin{aligned}
& \sum_{\substack{i, j=1 \\
i, j \text { even }}}^{b} \iint_{I_{i} \times I_{j}} K\left(\Psi_{i}(x), \Psi_{j}(y)\right) \operatorname{wal}_{k}(x) \overline{\operatorname{wal}_{k^{\prime}}(y)} d x d y \\
& = \begin{cases}\frac{1}{4} \widehat{K}(i, j) e^{-(2 \pi i / b)\left(k_{0}+k_{1}+\cdots+k_{n}\right)} e^{(2 \pi i / b)\left(k_{0}^{\prime}+k_{1}^{\prime}+\cdots+k_{n^{\prime}}^{\prime}\right)} \\
\text { if } \frac{b}{2}\left|k, \frac{b}{2}\right| k^{\prime}, i \oplus\left[\frac{k}{b}\right]=0, j \oplus\left[\frac{k^{\prime}}{b}\right]=0, \\
0 \quad \text { otherwise. }\end{cases}
\end{aligned}
$$

This gives the second term in the parenthesis (25).

Proof of (33). Starting with $K(x, y)=\sum_{i, j=0}^{\infty} \widehat{K}(i, j) \overline{\operatorname{wal}_{i}(x)} \operatorname{wal}_{j}(y)$ we find

$$
\begin{aligned}
& \int_{0}^{1} \int_{0}^{1} K(x, y) \overline{\operatorname{wal}_{[k / b]}(x)} \operatorname{wal}_{\left[k^{\prime} / b\right]}(y) d x d y \\
& =\sum_{i, j=0}^{\infty} \widehat{K}(i, j) \int_{0}^{1} \overline{\operatorname{wal}_{i}(x)} \overline{\operatorname{wal}_{[k / b]}(x)} d x \int_{0}^{1} \operatorname{wal}_{j}(y) \operatorname{wal}_{\left[k^{\prime} / b\right]}(y) d y .
\end{aligned}
$$

Because

$$
\operatorname{wal}_{j}(y) \operatorname{wal}_{\left[k^{\prime} / b\right]}(y)=\operatorname{wal}_{j \oplus\left[k^{\prime} / b\right]}(y)
$$

and if $\int_{0}^{1} \operatorname{wal}_{j}(y) \operatorname{wal}_{\left[k^{\prime} / b\right]}(y) d y \neq 0$, then $j \oplus\left[k^{\prime} / b\right]=0$.

Similarly, if $\int_{0}^{1} \overline{\operatorname{wal}_{i}(x)} \overline{\operatorname{wal}_{[k / b]}(x)} d x \neq 0$, then $i \oplus[k / b]=0$.

III) For odd $i$ and even $j$ we have

$$
\begin{aligned}
& \sum_{\substack{i, j=1 \\
i \text { odd }, j \text { even }}}^{b} \iint_{I_{i} \times I_{j}} K\left(\Phi_{i}(x), \Psi_{j}(y)\right) \operatorname{wal}_{k}(x) \overline{\operatorname{wal}_{k^{\prime}}(y)} d x d y \\
& \quad= \begin{cases}\frac{1}{4} \int_{0}^{1} \int_{0}^{1} K(x, y) \operatorname{wal}_{[k / b]}(x) \operatorname{wal}_{\left[k^{\prime} / b\right]}(y) e^{(2 \pi i / b)\left(k_{0}^{\prime}+k_{1}^{\prime}+\cdots+k_{n^{\prime}}^{\prime}\right)} d x d y & \text { if } \frac{b}{2}\left|k, \frac{b}{2}\right| k^{\prime}, \\
0 & \text { otherwise. }\end{cases}
\end{aligned}
$$

Now, using the Fourier-Walsh expansion $K(x, y)=\sum_{i, j=0}^{\infty} \widehat{K}(i, j) \overline{\operatorname{wal}_{i}(x)}$ wal $_{j}(y)$, we find

$$
\begin{aligned}
\int_{0}^{1} \int_{0}^{1} K(x, y) \mathrm{wal}_{[k / b]}(x) \mathrm{wal}_{\left[k^{\prime} / b\right]}(y) d x d y \\
\quad=\sum_{i, j=0}^{\infty} \widehat{K}(i, j) \int_{0}^{1} \overline{\operatorname{wal}_{i}(x)} \operatorname{wal}_{[k / b]}(x) d x \int_{0}^{1} \operatorname{wal}_{j}(y) \mathrm{wal}_{\left[k^{\prime} / b\right]}(y) d y \\
\quad= \begin{cases}\widehat{K}\left(\left[\frac{k}{b}\right], j\right) & \text { if } j \oplus\left[\frac{k^{\prime}}{b}\right]=0, \\
0 & \text { otherwise. }\end{cases}
\end{aligned}
$$

Proof of (36). If $\int_{0}^{1} \overline{\operatorname{wal}_{i}(x)} \operatorname{wal}_{[k / b]}(x) d x \neq 0$, then $i=[k / b]$. If $\int_{0}^{1} \operatorname{wal}_{j}(y) \operatorname{wal}_{\left[k^{\prime} / b\right]}(y) d y \neq 0$, then $j \oplus\left[k^{\prime} / b\right]=0$. 
Thus (35) is equal to

$$
\begin{cases}\frac{1}{4} \widehat{K}\left(\left[\frac{k}{b}\right], j\right) e^{(2 \pi i / b)\left(k_{0}^{\prime}+k_{1}^{\prime}+\cdots+k_{n^{\prime}}^{\prime}\right)} & \text { if } \frac{b}{2}\left|k, \frac{b}{2}\right| k^{\prime}, j \oplus\left[\frac{k^{\prime}}{b}\right]=0 \\ 0 & \text { otherwise. }\end{cases}
$$

IV) Similarly, for even $i$ and odd $j$ we have

$$
\begin{aligned}
& \sum_{\substack{i, j=1 \\
i \text { even, } j \text { odd }}}^{b} \iint_{I_{i} \times I_{j}} K\left(\Psi_{i}(x), \Psi_{j}(y)\right) \mathrm{wal}_{k}(x) \overline{\mathrm{wal}_{k^{\prime}}(y)} d x d y \\
& \quad= \begin{cases}\frac{1}{4} \widehat{K}\left(i,\left[\frac{k^{\prime}}{b}\right]\right) e^{-(2 \pi i / b)\left(k_{0}+k_{1}+\cdots+k_{n}\right)} & \text { if } \frac{b}{2}\left|k, \frac{b}{2}\right| k^{\prime}, i \oplus\left[\frac{k}{b}\right]=0, \\
0 & \text { otherwise. }\end{cases}
\end{aligned}
$$

Summing up (29), (34), (37), and (38) we find

$$
\begin{gathered}
\widehat{K}_{1}\left(k, k^{\prime}\right)=\frac{1}{4}\left(\widehat{K}\left(\left[\frac{k}{b}\right],\left[\frac{k^{\prime}}{b}\right]\right)+\widehat{K}\left(-\left[\frac{k}{b}\right],-\left[\frac{k^{\prime}}{b}\right]\right)+\widehat{K}\left(\left[\frac{k}{b}\right],-\left[\frac{k^{\prime}}{b}\right]\right) e^{(2 \pi i / b)\left(k_{0}^{\prime}+k_{1}^{\prime}+\cdots+k_{n}^{\prime}\right)}\right. \\
\left.+\widehat{K}\left(-\left[\frac{k}{b}\right],\left[\frac{k^{\prime}}{b}\right]\right) e^{-(2 \pi i / b)\left(k_{0}+k_{1}+\cdots+k_{n}\right)}\right)
\end{gathered}
$$

Inserting (39) into (12) we find (25).

Now, the proof of Theorem 5 is complete.

For some control of a Fourier-Walsh expansion of a kernel $K(\mathbf{x}, \mathbf{y})$, the following theorem can be used.

TheOREM 6. For an arbitrary u.d.p. map $\Psi(\mathbf{x}, \boldsymbol{\sigma})$ and an arbitrary kernel $K(\mathbf{x}, \mathbf{y})$, for $N=1$ we have

$$
\int_{[0,1]^{s}} \sup _{\substack{f \in H \\\|f\| \leqslant 1}}\left|f\left(\Psi\left(\mathbf{x}_{0}, \boldsymbol{\sigma}\right)\right)-\int_{[0,1]^{s}} f(\mathbf{x}) d \mathbf{x}\right|^{2} d \boldsymbol{\sigma}=\int_{[0,1]^{s}} K(\mathbf{x}, \mathbf{x}) d \mathbf{x}-\int_{[0,1]^{2 s}} K(\mathbf{x}, \mathbf{y}) d \mathbf{x} d \mathbf{y} .
$$

Proof. For $N=1$, the equation (7) implies

$$
\begin{aligned}
\int_{[0,1]^{s}} & \sup _{\substack{f \in H \\
\|f\| \leqslant 1}}\left|f\left(\Psi\left(\mathbf{x}_{0}, \boldsymbol{\sigma}\right)\right)-\int_{[0,1]^{s}} f(\mathbf{x}) d \mathbf{x}\right|^{2} d \boldsymbol{\sigma} \\
= & \int_{[0,1]^{s}} K\left(\Psi\left(\mathbf{x}_{0}, \boldsymbol{\sigma}\right), \Psi\left(\mathbf{x}_{0}, \boldsymbol{\sigma}\right)\right) d \boldsymbol{\sigma}-\int_{[0,1]^{2 s}} K(\mathbf{x}, \mathbf{y}) d \mathbf{x} d \mathbf{y} \\
= & \int_{[0,1]^{s}} K(\mathbf{x}, \mathbf{x}) d \mathbf{x}-\int_{[0,1]^{2 s}} K(\mathbf{x}, \mathbf{y}) d \mathbf{x} d \mathbf{y} .
\end{aligned}
$$

REMARK 2. Theorem 6 implies the following.

By Theorem 2 and Theorem 3 we have that (40) is equal to

$$
\sum_{\substack{\mathbf{k} \in \mathbb{N}_{0}^{s} \\ \mathbf{k} \neq \mathbf{0}}} \widehat{K}(\mathbf{k}, \mathbf{k})=\sum_{\substack{\mathbf{k} \in \mathbb{N}_{0}^{s} \\ \mathbf{k} \neq \mathbf{0}}} \widehat{K}_{1}(\mathbf{k}, \mathbf{k})
$$

for an arbitrary u.d.p. map $\Phi(\mathbf{x})$ and a common kernel $K(\mathbf{x}, \mathbf{y})$. 
By Theorem 4 we have

$$
\sum_{\substack{\mathbf{k} \in \mathbb{N}_{0}^{s} \\ \mathbf{k} \neq \mathbf{0}}} \widehat{K}(\mathbf{k}, \mathbf{k})=\sum_{\substack{\mathbf{k} \in \mathbb{N}_{0}^{s} \\ \mathbf{k} \neq \mathbf{0}, \mathbf{b}^{\alpha} \mid \mathbf{k}}} \widehat{K}\left(\frac{\mathbf{k}}{\mathbf{b}^{\boldsymbol{\alpha}}}, \frac{\mathbf{k}}{\mathbf{b}^{\boldsymbol{\alpha}}}\right)
$$

for $\Phi(\mathbf{x})=\mathbf{b}^{\boldsymbol{\alpha}} \mathbf{x} \bmod 1 .^{12}$

By Theorem 5, for one-dimensional tent map $\Phi(x)$, we have (41) of the form

$$
\begin{aligned}
& \sum_{k=1}^{\infty} \widehat{K}(k, k) \\
& =\sum_{\substack{k=1 \\
(b / 2) \mid k}}^{\infty} \frac{1}{4}\left(\widehat{K}\left(\left[\frac{k}{b}\right],\left[\frac{k}{b}\right]\right)+\widehat{K}\left(-\left[\frac{k}{b}\right],-\left[\frac{k}{b}\right]\right)+\widehat{K}\left(\left[\frac{k}{b}\right],-\left[\frac{k}{b}\right]\right) e^{(2 \pi i / b)\left(k_{0}+k_{1}+\cdots+k_{n}\right)}\right. \\
& \left.+\widehat{K}\left(-\left[\frac{k}{b}\right],\left[\frac{k}{b}\right]\right) e^{-(2 \pi i / b)\left(k_{0}+k_{1}+\cdots+k_{n}\right)}\right) .
\end{aligned}
$$

Another proof of (43). Let $k=k_{0}+k_{1} b+\cdots+k_{n} b^{n}, k_{n} \neq 0$. For $(b / 2) \mid k$ we have $k_{0}=0$ or $k_{0}=b / 2$. Put

$$
A=\left\{0+\left[\frac{k}{b}\right] b, \frac{b}{2}+\left[\frac{k}{b}\right] b\right\}
$$

Then

$$
\begin{gathered}
\sum_{k \in A} \widehat{K}\left(\left[\frac{k}{b}\right],\left[\frac{k}{b}\right]\right)=2 \widehat{K}\left(\left[\frac{k}{b}\right],\left[\frac{k}{b}\right]\right), \\
\sum_{k \in A} \widehat{K}\left(-\left[\frac{k}{b}\right],-\left[\frac{k}{b}\right]\right)=2 \widehat{K}\left(-\left[\frac{k}{b}\right],-\left\lceil\frac{k}{b}\right]\right), \\
\sum_{k \in A} \widehat{K}\left(\left[\frac{k}{b}\right],-\left[\frac{k}{b}\right]\right) e^{(2 \pi i / b)\left(k_{0}+k_{1}+\cdots+k_{n}\right)} \\
=\widehat{K}\left(\left[\frac{k}{b}\right],-\left[\frac{k}{b}\right]\right) e^{(2 \pi i / b)\left(k_{1}+\cdots+k_{n}\right)}\left(e^{(2 \pi i / b) 0}+e^{(2 \pi i / b)(b / 2)}\right)=0, \\
\sum_{k \in A} \widehat{K}\left(-\left[\frac{k}{b}\right],\left[\frac{k}{b}\right]\right) e^{-(2 \pi i / b)\left(k_{0}+k_{1}+\cdots+k_{n}\right)}=0 .
\end{gathered}
$$

\section{Numerical results}

4.1. The mean square worst-case error for the $\operatorname{kernel} K(x, y)=1-\max (x, y)$.

In this Section we apply Theorems 2, 3, 4 and 5 to the Fourier-Walsh expansion of the simple kernel $K(x, y)=1-\max (x, y)$.

THEOREM 7. Let $K(x, y)=\sum_{k, k^{\prime} \in \mathbb{N}_{0}} \widehat{K}\left(k, k^{\prime}\right) \overline{\mathrm{wal}_{k}(x)} \mathrm{wal}_{k^{\prime}}(y)$ be the Fourier-Walsh series of the kernel $K(x, y)=1-\max (x, y)$. Then for $k \neq 0, k=k_{0}+k_{1} b+\cdots+k_{n} b^{n}, k_{n} \neq 0$, we have

$$
\widehat{K}(k, k)=-\frac{1}{2} \frac{1}{b^{2(n+1)}}\left(\frac{1}{3}-\frac{1}{\sin ^{2}\left(k_{n}(\pi / b)\right)}\right),
$$

\footnotetext{
${ }^{12}$ This holds for an arbitrary function $\widehat{K}(\mathbf{k}, \mathbf{k})$ directly.
} 
and

$$
\widehat{K}(k,-k)= \begin{cases}0 & \text { if not } 2 k_{0}=\cdots=2 k_{n-1}=0(\bmod b), \\ \frac{1}{3 b^{2(n+1)}} & \text { if } k_{n}=\frac{b}{2}, 2 k_{0}=\cdots=2 k_{n-1}=0(\bmod b), \\ \frac{1}{b^{2(n+1)}\left(e^{(2 \pi / b) k_{n}}-1\right)^{2}} & \text { if } k_{n} \neq \frac{b}{2}, 2 k_{0}=\cdots=2 k_{n-1}=0(\bmod b) .\end{cases}
$$

Proof of (44). Let the kernel $K(x, y)$ be rewritten in the form

$$
K(x, y)=1-\frac{x+y+|x-y|}{2} .
$$

By $[5 ;(12.15)]$ for $k=k_{0}+k_{1} b+\cdots+k_{n} b^{n}$ we have

$$
\int_{0}^{1} \int_{0}^{1}|x-y| \operatorname{wal}_{k}(x) \overline{\operatorname{wal}_{k}(y)} d x d y=\frac{1}{b^{2(n+1)}}\left(\frac{1}{3}-\frac{1}{\sin ^{2}\left(k_{n}(\pi / b)\right)}\right) .
$$

For $k \neq 0$ we have

$$
\int_{0}^{1} \int_{0}^{1} x \operatorname{wal}_{k}(x) \overline{\operatorname{wal}_{k}(y)} d x d y=0
$$

then (47) implies (44).

Proof of (45). We have

$$
\begin{aligned}
\widehat{K}(k,-k) & =\int_{0}^{1} \int_{0}^{1} K(x, y) \operatorname{wal}_{k}(x) \overline{\mathrm{wal}_{-k}(y)} d x d y \\
& =\int_{0}^{1} \int_{0}^{1} K(x, y) \operatorname{wal}_{k}(x) \operatorname{wal}_{k}(y) d x d y=-\frac{1}{2} \int_{0}^{1} \int_{0}^{1}|x-y| \operatorname{wal}_{k}(x) \operatorname{wal}_{k}(y) d x d y .
\end{aligned}
$$

Now, $k=k_{0}+k_{1} b+\cdots+k_{n} b^{n}, k_{n} \neq 0$,

$$
\begin{gathered}
I_{i}=\left[\frac{i}{b^{n+1}}, \frac{i+1}{b^{n+1}}\right), \quad i=0,1,2, \ldots, b^{n+1}-1, \\
x=\frac{x_{0}}{b}+\frac{x_{1}}{b^{2}}+\cdots+\frac{x_{n}}{b^{n+1}}+\cdots \in I_{i}, \quad \frac{i}{b^{n+1}}=\frac{x_{0}}{b}+\frac{x_{1}}{b^{2}}+\cdots+\frac{x_{n}}{b^{n+1}}, \\
y=\frac{y_{0}}{b}+\frac{y_{1}}{b^{2}}+\cdots+\frac{y_{n}}{b^{n+1}}+\cdots \in I_{j}, \quad \frac{j}{b^{n+1}}=\frac{y_{0}}{b}+\frac{y_{1}}{b^{2}}+\cdots+\frac{y_{n}}{b^{n+1}} .
\end{gathered}
$$

Using these notations we continue to calculate (48).

$$
\begin{aligned}
& -\frac{1}{2} \int_{0}^{1} \int_{0}^{1}|x-y| \operatorname{wal}_{k}(x) \operatorname{wal}_{k}(y) d x d y \\
& \quad=-\frac{1}{2} \sum_{i, j=0}^{b^{n+1}-1} e^{(2 \pi i / b)\left(\left(x_{0}+y_{0}\right) k_{0}+\left(x_{1}+y_{1}\right) k_{1}+\cdots+\left(x_{n}+y_{n}\right) k_{n}\right)} \iint_{I_{i} \times I_{j}}|x-y| d x d y,
\end{aligned}
$$

where

$$
\iint_{I_{i} \times I_{j}}|x-y| d x d y= \begin{cases}\frac{1}{3 b^{3(n+1)}} & \text { if } i=j \\ \frac{|i-j|}{b^{3(n+1)}} & \text { if } i \neq j\end{cases}
$$


I) For $i=j$ in (49) we have

$$
\begin{aligned}
& -\frac{1}{2} \frac{1}{3 b^{3(n+1)}} \sum_{i=0}^{b^{n+1}-1} e^{(2 \pi i / b)\left(2 x_{0} k_{0}+2 x_{1} k_{1}+\cdots+2 x_{n} k_{n}\right)} \\
& \quad=-\frac{1}{2} \frac{1}{3 b^{3(n+1)}}\left(\sum_{x_{0}=0}^{b-1} e^{(2 \pi i / b) 2 x_{0} k_{0}}\right)\left(\sum_{x_{1}=0}^{b-1} e^{(2 \pi i / b) 2 x_{1} k_{1}}\right) \cdots\left(\sum_{x_{n}=0}^{b-1} e^{(2 \pi i / b) 2 x_{n} k_{n}}\right) \\
& \quad= \begin{cases}-\frac{1}{2} \frac{1}{3 b^{2(n+1)}} & \text { if } 2 k_{0}=2 k_{1}=\cdots=2 k_{n}=0(\bmod b), \\
0, & \text { otherwise. }\end{cases}
\end{aligned}
$$

II) For $i \neq j$ in (49) we have

$$
\begin{gathered}
-\frac{1}{2} \frac{2}{b^{2(n+1)}} \sum_{j=0}^{b^{n+1}-2} \sum_{i=j+1}^{b^{n+1}-1}\left(\frac{i}{b^{n+1}}-\frac{j}{b^{n+1}}\right) e^{(2 \pi i / b)\left(\left(x_{0}+y_{0}\right) k_{0}+\left(x_{1}+y_{1}\right) k_{1}+\cdots+\left(x_{n}+y_{n}\right) k_{n}\right)} \\
=-\frac{1}{2} \frac{2}{b^{2(n+1)}} \sum_{j=0}^{b^{n+1}-2} \sum_{i=j+1}^{b^{n+1}-1}\left(\frac{x_{0}-y_{0}}{b}+\frac{x_{1}-y_{1}}{b^{2}}+\cdots+\frac{x_{n}-y_{n}}{b^{n+1}}\right) \\
\times e^{(2 \pi i / b)\left(\left(x_{0}+y_{0}\right) k_{0}+\left(x_{1}+y_{1}\right) k_{1}+\cdots+\left(x_{n}+y_{n}\right) k_{n}\right)} .
\end{gathered}
$$

Now, for fixed $x_{0}, x_{1}, \ldots, x_{n-1}$ and $y_{0}, y_{1}, \ldots, y_{n-1}$, we assume that

$$
\frac{x_{0}-y_{0}}{b}+\frac{x_{1}-y_{1}}{b^{2}}+\cdots+\frac{x_{n-1}-y_{n-1}}{b^{n}}>0 .
$$

Similarly, as in $[5 ;(12.11)]$ the following partial sum of $(52)$ is zero.

$$
\begin{gathered}
\left|\sum_{x_{n}=0}^{b-1} \sum_{y_{n}=0}^{b-1}\left(\frac{x_{0}-y_{0}}{b}+\cdots+\frac{x_{n}-y_{n}}{b^{n+1}}\right) e^{(2 \pi i / b)\left(\left(x_{0}+y_{0}\right) k_{0}+\cdots+\left(x_{n}+y_{n}\right) k_{n}\right)}\right| \\
=\left|\sum_{x_{n}=0}^{b-1} \sum_{y_{n}=0}^{b-1}\left(\frac{x_{0}-y_{0}}{b}+\cdots+\frac{x_{n}-y_{n}}{b^{n+1}}\right) e^{(2 \pi i / b)\left(x_{n}+y_{n}\right) k_{n}}\right| \\
=\mid\left(\frac{x_{0}-y_{0}}{b}+\cdots+\frac{x_{n-1}-y_{n-1}}{b^{n}}\right) \sum_{x_{n}=0}^{b-1} \sum_{y_{n}=0}^{b-1} e^{(2 \pi i / b)\left(x_{n}+y_{n}\right) k_{n}} \\
\quad+\sum_{x_{n}=0}^{b-1} \sum_{y_{n}=0}^{b-1} \frac{\left(x_{n}-y_{n}\right)}{b^{n+1}} e^{(2 \pi i / b)\left(x_{n}+y_{n}\right) k_{n}} \mid=0,
\end{gathered}
$$

since the final double sums in (54) are equal to zero, bearing in mind $k_{n} \neq 0$.

III) Note that from (53) follows that

$$
\frac{x_{0}-y_{0}}{b}+\frac{x_{1}-y_{1}}{b^{2}}+\cdots+\frac{x_{n}-y_{n}}{b^{n}}>0
$$

for arbitrary $x_{n}, y_{n}=0,1, \ldots, b-1$. Thus it remains to consider the case

$$
\frac{x_{0}-y_{0}}{b}+\frac{x_{1}-y_{1}}{b^{2}}+\cdots+\frac{x_{n-1}-y_{n-1}}{b^{n}}=0,
$$


i.e., $x_{0}=y_{0}, x_{1}=y_{1}, \ldots, x_{n-1}=y_{n-1}$, but in this case for $j<i$ we need $y_{n}<x_{n}$. For such subsum we have

$$
\begin{aligned}
& \sum_{j=0}^{b^{n+1}-2} \sum_{i=j+1}^{b^{n+1}-1}\left(\frac{x_{0}-y_{0}}{b}+\cdots+\frac{x_{n}-y_{n}}{b^{n+1}}\right) e^{(2 \pi i / b)\left(\left(x_{0}+y_{0}\right) k_{0}+\cdots+\left(x_{n}+y_{n}\right) k_{n}\right)} \\
& =\sum_{x_{0}=0}^{b-1} \cdots \sum_{x_{n-1}=0}^{b-1} \sum_{y_{n}=0}^{b-2} \sum_{x_{n}=y_{n}+1}^{b-1}\left(\frac{x_{n}-y_{n}}{b^{n+1}}\right) e^{(2 \pi i / b)\left(2 x_{0} k_{0}+\cdots+2 x_{n-1} k_{n-1}+\left(x_{n}+y_{n}\right) k_{n}\right)} \\
& =\left(\sum_{x_{0}=0}^{b-1} e^{(2 \pi i / b) 2 x_{0} k_{0}}\right) \cdots\left(\sum_{x_{n-1}=0}^{b-1} e^{\left.(2 \pi i / b) 2 x_{n-1} k_{n-1}\right)}\right. \\
& = \begin{cases}b^{n} \sum_{y_{n}=0}^{b-2} \sum_{y_{n}=0}^{b-1}\left(\frac{x_{n}=y_{n}+1}{b-1}\left(\frac{x_{n}-y_{n}}{b^{n+1}}\right) e^{(2 \pi i / b)\left(x_{n}+y_{n}\right) k_{n}}\right. \\
0 \quad \text { if } 2 k_{0}=\cdots=2 k_{n-1}=0(\bmod b),\end{cases}
\end{aligned}
$$

Replacing $x_{n}-y_{n}=j$, the final double sum in (56) can be expressed as

$$
\sum_{y_{n}=0}^{b-2} e^{(2 \pi i / b) 2 y_{n} k_{n}} \sum_{j=1}^{b-1-y_{n}} \frac{j}{b^{n+1}} e^{(2 \pi i / b) j k_{n}} .
$$

Then we use the following summation formula

$$
\sum_{j=1}^{n} j e^{B j}=\frac{n-1}{1-e^{-B .1}}-\frac{e^{B(n-1)}-1}{\left(1-e^{-B .1}\right)^{2}}+n \frac{e^{B n}-1}{1-e^{-B .1}}
$$

which holds for $n=0,1,2, \ldots$ and for an arbitrary nonzero complex number $B$. Inserting (58) into (57) we find

$$
\begin{aligned}
& \sum_{y_{n}=0}^{b-2} \sum_{x_{n}=y_{n}+1}^{b-1}\left(\frac{x_{n}-y_{n}}{b^{n+1}}\right) e^{(2 \pi i / b)\left(x_{n}+y_{n}\right) k_{n}} \\
& =\frac{1}{b^{n+1}} \frac{e^{-(2 \pi i / b) k_{n}}}{\left(1-e^{-(2 \pi i / b) k_{n}}\right)^{2}} \sum_{y_{n}=0}^{b-2} e^{(2 \pi i / b) 2 y_{n} k_{n}} \\
& \quad+\frac{1}{b^{n+1}}\left((b-1) \frac{e^{-(2 \pi i / b) k_{n}}}{1-e^{-(2 \pi i / b) k_{n}}}-\frac{e^{-(4 \pi i / b) k_{n}}}{\left(1-e^{-(2 \pi i / b) k_{n}}\right)^{2}}\right) \sum_{y_{n}=0}^{b-2} e^{(2 \pi i / b) y_{n} k_{n}} \\
& \quad-\frac{1}{b^{n+1}} \frac{e^{-(2 \pi i / b) k_{n}}}{1-e^{-(2 \pi i / b) k_{n}}} \sum_{y_{n}=0}^{b-2} y_{n} e^{(2 \pi i / b) y_{n} k_{n}} .
\end{aligned}
$$

Here

$$
\sum_{y_{n}=0}^{b-2} e^{(2 \pi i / b) 2 y_{n} k_{n}}= \begin{cases}b-1 & \text { if } k_{n}=\frac{b}{2} \\ -e^{-(4 \pi i / b) k_{n}} & \text { if } k_{n} \neq \frac{b}{2}\end{cases}
$$




$$
\begin{aligned}
\sum_{y_{n}=0}^{b-2} e^{(2 \pi i / b) y_{n} k_{n}} & =-e^{-(2 \pi i / b) k_{n}}, \\
\sum_{y_{n}=0}^{b-2} y_{n} e^{(2 \pi i / b) y_{n} k_{n}} & =\frac{-1}{1-e^{-(2 \pi i / b) k_{n}}}-\frac{e^{-(6 \pi i / b) k_{n}}-1}{\left(1-e^{-(2 \pi i / b) k_{n}}\right)^{2}}+(b-1) \frac{e^{-(4 \pi i / b) k_{n}}}{1-e^{-(2 \pi i / b) k_{n}}} .
\end{aligned}
$$

Inserting expression (60) into (59) we find

$$
\sum_{y_{n}=0}^{b-2} \sum_{x_{n}=y_{n}+1}^{b-1}\left(\frac{x_{n}-y_{n}}{b^{n+1}}\right) e^{(2 \pi i / b)\left(x_{n}+y_{n}\right) k_{n}}=\frac{1}{b^{n+1}} \begin{cases}\frac{-b e^{-(4 \pi i / b) k_{n}}}{\left(1-e^{-(2 \pi i / b) k_{n}}\right)^{2}} & \text { if } k_{n} \neq \frac{b}{2} \\ \frac{(b-2) e^{-(2 \pi i / b) k_{n}}}{1-e^{-(2 \pi i / b) k_{n}}}+e^{-(2 \pi i / b) k_{n}} & \text { if } k_{n}=\frac{b}{2} .\end{cases}
$$

For $k_{n}=b / 2$ the relevant part of $(61)$ is equal to $-b / 2$. Finally, summing up (51), (56) and (61) we find (45).

This completes the proof of Theorem 7.

THEOREM 8. Let $b \geqslant 2, \alpha$ and $\beta$ be integer numbers, $\alpha<\beta$, and

(i) $K(x, y)=1-\max (x, y)$;

(ii) $N=b^{\beta}$;

(iii) $x_{0}, x_{1}, \ldots, x_{N-1}$ be the van der Corput sequence in base $b .^{13}$

Then

$$
\begin{gathered}
\int_{0}^{1} \sup _{\substack{f \in H \\
\|f\| \leqslant 1}}\left|\frac{1}{N} \sum_{n=0}^{N-1} f\left(x_{n} \oplus \sigma\right)-\int_{0}^{1} f(x) d x\right|^{2} d \sigma=\frac{1}{6 b^{2 \beta}}, \\
\int_{0}^{1} \sup _{\substack{f \in H \\
\|f\| \leqslant 1}}\left|\frac{1}{N} \sum_{n=0}^{N-1} f\left(\Phi\left(x_{n} \oplus \sigma\right)\right)-\int_{0}^{1} f(x) d x\right|^{2} d \sigma=\frac{1}{6 b^{2(\beta-\alpha)}}
\end{gathered}
$$

for $\Phi(x)=b^{\alpha} x \bmod 1$ and $\alpha<\beta$

$$
\int_{0}^{1} \sup _{\substack{f \in H \\\|f\| \leqslant 1}}\left|\frac{1}{N} \sum_{n=0}^{N-1} f\left(\Phi\left(x_{n} \oplus \sigma\right)\right)-\int_{0}^{1} f(x) d x\right|^{2} d \sigma=\frac{1}{24 b^{2(\beta-1)}}
$$

for the tent function $\Phi(x), 2 \mid b$.

Proof of (62) By (10), in one-dimensional case, the left hand side of (62) is equal to

$$
\sum_{k=1}^{\infty} \widehat{K}(k, k)\left|\frac{1}{N} \sum_{n=0}^{N-1} \mathrm{wal}_{k}\left(x_{n}\right)\right|^{2}
$$

For $N=b^{\beta}$ and for the van der Corput sequence $x_{n}$ we have

$$
\frac{1}{N} \sum_{n=0}^{N-1} \operatorname{wal}_{k}\left(x_{n}\right)= \begin{cases}0 & \text { if } b^{\beta} \nmid k \\ 1 & \text { if } b^{\beta} \mid k\end{cases}
$$

${ }^{13}$ This is the same sequence as

$$
\frac{0}{b^{\beta}}, \quad \frac{1}{b^{\beta}}, \quad \ldots, \quad \frac{b^{\beta}-1}{b^{\beta}} .
$$


Inserting (44) into (65) we find

$$
\sum_{k=1, b^{\beta} \mid k}^{\infty} \widehat{K}(k, k)=-\frac{1}{2} \sum_{n=\beta}^{\infty} \frac{1}{b^{2(n+1)}} b^{n-\beta} \sum_{k_{n}=1}^{b-1}\left(\frac{1}{3}-\frac{1}{\sin ^{2}\left(k_{n}(\pi / b)\right)}\right) .
$$

Here the term $b^{n-\beta}$ is the number of $k, b^{\beta} \mid k$, for which

$$
k_{\beta}=0,1, \ldots, b-1, \quad k_{\beta+1}=0,1, \ldots, b-1, \quad \ldots, \quad k_{n-1}=0,1, \ldots, b-1 .
$$

Since [5; Corollary A23]

$$
\sum_{k_{n}=1}^{b-1} \frac{1}{\sin ^{2}\left(k_{n}(\pi / b)\right)}=\frac{b^{2}-1}{3}
$$

we find (62).

Here we add

Proof of (66). Let $N=b^{\beta}$ and $x_{0}, x_{1}, \ldots, x_{N-1}$ be the van der Corput sequence,

$$
\begin{gathered}
n=n_{0}+n_{1} b+\cdots+n_{\beta-1} b^{\beta-1}, \quad n_{i}=0,1, \ldots, b-1, \\
x_{n}=\frac{n_{0}}{b}+\frac{n_{1}}{b}+\cdots+\frac{n_{\beta-1}}{b^{\beta}}, \quad k=k_{0}+k_{1} b+k_{2} b^{2}+\cdots .
\end{gathered}
$$

We have

$$
\sum_{n=0}^{b^{\beta}-1} \operatorname{wal}_{k}\left(x_{n}\right)=\left(\sum_{n_{0}=0}^{b-1} e^{(2 \pi i / b) n_{0} k_{0}}\right)\left(\sum_{n_{1}=0}^{b-1} e^{(2 \pi i / b) n_{1} k_{1}}\right) \cdots\left(\sum_{n_{\beta-1}=0}^{b-1} e^{(2 \pi i / b) n_{\beta-1} k_{\beta-1}}\right) .
$$

From this if $k_{i} \neq 0$ for some $0 \leqslant i \leqslant \beta-1$, then $\sum_{n=0}^{b^{\beta}-1}$ wal $_{k}\left(x_{n}\right)=0$. If $k_{0}=k_{1}=\cdots=k_{\beta-1}=0$, i.e. $b^{\beta} \mid k$, then $\left(1 / b^{\beta}\right) \sum_{n=0}^{b^{\beta}-1} \operatorname{wal}_{k}\left(x_{n}\right)=1 .^{14}$

Proof of (63). By one-dimensional case of (13) of Theorem 4 the mean square worst-case error (63) is

$$
\sum_{k=1, b^{\alpha} \mid k}^{\infty} \widehat{K}\left(\frac{k}{b^{\alpha}}, \frac{k}{b^{\alpha}}\right)\left|\frac{1}{N} \sum_{n=0}^{N-1} \mathrm{wal}_{k}\left(x_{n}\right)\right|^{2} .
$$

By (66) the sum (69) is equal to

$$
\sum_{k=1, b^{\alpha}\left|k, b^{\beta}\right| k}^{\infty} \widehat{K}\left(\frac{k}{b^{\alpha}}, \frac{k}{b^{\alpha}}\right)
$$

Assuming $\alpha<\beta$,

$$
k=k_{\beta}+\cdots+k_{n} b^{n}, \quad \frac{k}{b^{\alpha}}=k_{\beta} b^{\beta-\alpha}+\cdots+k_{n} b^{n-\alpha},
$$

then (70) has the form

$$
\sum_{k=1, b^{\beta} \mid k}^{\infty} \widehat{K}\left(\frac{k}{b^{\alpha}}, \frac{k}{b^{\alpha}}\right)=-\frac{1}{2} \sum_{n=\beta}^{\infty} \frac{1}{b^{2(n-\alpha+1)}} b^{n-\beta} \sum_{k_{n}=1}^{b-1}\left(\frac{1}{3}-\frac{1}{\sin ^{2}\left(k_{n}(\pi / b)\right)}\right),
$$

which gives (63). Here the exponent in $b^{2(n-\alpha+1)}$ is given by (44) after transformation $k \rightarrow k / b^{\alpha}$.

\footnotetext{
${ }^{14}$ For an arbitrary $N$, see $(88)$.
} 
REMARK 3. The formula (63) follows also from the second expression (14) in Theorem 4 using

$$
\frac{1}{b^{\beta}} \sum_{n=0}^{b^{\beta}-1} \operatorname{wal}_{k}\left(\Phi\left(x_{n}\right)\right)= \begin{cases}0 & \text { if } b^{\beta-\alpha} \nmid k \\ 1 & \text { if } b^{\beta-\alpha} \mid k\end{cases}
$$

Proof of (64). We start with

$$
\sum_{\substack{k=1 \\ k=k_{\beta} b^{\beta}+\cdots+k_{n} b^{n}}}^{\infty} \widehat{K}\left(\frac{k}{b}, \frac{k}{b}\right)=\sum_{\substack{k=1 \\ k=k_{\beta} b^{\beta}+\cdots+k_{n} b^{n}}}^{\infty} \widehat{K}\left(-\frac{k}{b},-\frac{k}{b}\right)=\frac{1}{6 b^{2(\beta-1)}}
$$

and

$$
\begin{aligned}
\sum_{\substack{k=1 \\
k=k_{\beta} b^{\beta}+\cdots+k_{n} b^{n}}}^{\infty} \widehat{K}\left(\frac{k}{b},-\frac{k}{b}\right) e^{(2 \pi i / b)\left(k_{\beta}+\cdots+k_{n}\right)} & =\sum_{\substack{k=1 \\
k=k_{\beta} b^{\beta}+\cdots+k_{n} b^{n}}}^{\infty} \widehat{K}\left(-\frac{k}{b}, \frac{k}{b}\right) e^{-(2 \pi i / b)\left(k_{\beta}+\cdots+k_{n}\right)} \\
& =-\frac{1}{12 b^{2(\beta-1)}}
\end{aligned}
$$

Inserting (73) and (74) into (25) then by Theorem 5 the mean square worst-case error is

$$
=\frac{1}{4}\left(\frac{1}{6 b^{2(\beta-1)}}+\frac{1}{6 b^{2(\beta-1)}}-\frac{1}{12 b^{2(\beta-1)}}-\frac{1}{12 b^{2(\beta-1)}}\right)=\frac{1}{24 b^{2(\beta-1)}},
$$

which gives (64).

Proof of (74). By $(45)$, if $2 k_{\beta}=\cdots=2 k_{n-1}=0(\bmod b)$, then the value of $\widehat{K}(k / b,-k / b)$ depends only on $k_{n}$, thus

$$
\begin{aligned}
& \sum_{\substack{k=1 \\
k=k_{\beta} b^{\beta}+\cdots+k_{n} b^{n}}}^{\infty} \widehat{K}\left(\frac{k}{b},-\frac{k}{b}\right) e^{(2 \pi i / b)\left(k_{\beta}+\cdots+k_{n}\right)} \\
= & \sum_{n=\beta}^{\infty} \sum_{k_{\beta}=0, b / 2, \ldots, k_{n-1}=0, b / 2} \widehat{K}\left(\frac{k}{b},-\frac{k}{b}\right) e^{(2 \pi i / b)\left(k_{\beta}+\cdots+k_{n}\right)} \\
= & \left(\sum_{k_{\beta}=0, b / 2} e^{(2 \pi i / b) k_{\beta}}\right) \cdots\left(\sum_{k_{n-1}=0, b / 2} e^{(2 \pi i / b) k_{n-1}}\right) \sum_{k_{n}=1}^{b-1} \widehat{K}\left(\frac{k}{b},-\frac{k}{b}\right) e^{(2 \pi i / b) k_{n}}
\end{aligned}
$$

and every sum in parenthesis is equal to zero. But for $n=\beta$ the set of such parenthesis is empty and by using (45) we have that (75) is equal

$$
\sum_{k_{n}=1}^{b-1} \widehat{K}\left(\frac{k}{b},-\frac{k}{b}\right) e^{(2 \pi i / b) k_{n}}=-\frac{1}{3 b^{2 \beta}}+\sum_{k_{n}=1, k_{n} \neq b / 2}^{b-1} \frac{e^{(2 \pi i / b) k_{n}}}{\left(e^{(2 \pi i / b) k_{n}}-1\right)^{2}} .
$$

Note that we apply (45) for $k / b=k_{\beta} b^{\beta}+\cdots+k_{n} b^{n-1}$ and for $n=\beta$ we have $1 / b^{2(n-1+1)}=1 / b^{2 \beta}$. Moreover, by elementary operations (all the time $k_{n} \neq 0$ )

$$
\frac{e^{(2 \pi i / b) k_{n}}}{\left(e^{(2 \pi i / b) k_{n}}-1\right)^{2}}=-\frac{1}{4 \sin ^{2}\left((\pi / b) k_{n}\right)} .
$$

Using (68) we find

$$
\sum_{k_{n}=1}^{b-1} \frac{e^{(2 \pi i / b) k_{n}}}{\left(e^{(2 \pi i / b) k_{n}}-1\right)^{2}}=-\frac{1}{12}\left(b^{2}-1\right) .
$$


For the sum in (76) we need to omit in (77) the term for $k_{n}=b / 2$, which is $-1 /(-1-1)^{2}=-1 / 4$. Summary,

$$
\sum_{\substack{k=1 \\ k=k_{\beta} b^{\beta}+\cdots+k_{n} b^{n}}}^{\infty} \widehat{K}\left(\frac{k}{b},-\frac{k}{b}\right) e^{(2 \pi i / b)\left(k_{\beta}+\cdots+k_{n}\right)}=-\frac{1}{3 b^{2 \beta}}-\frac{1}{4 b^{2 \beta}} \frac{b^{2}-1}{3}+\frac{1}{4 b^{2 \beta}}=-\frac{1}{12 b^{2(\beta-1)}} .
$$

Final notes. By definition of Fourier-Walsh coefficients we have

$$
\begin{aligned}
& \widehat{K}(k,-k)=\int_{0}^{1} \int_{0}^{1} K(x, y) \mathrm{wal}_{k}(x) \overline{\mathrm{wal}_{-k}(y)} d x d y=\int_{0}^{1} \int_{0}^{1} K(x, y) \operatorname{wal}_{k}(x) \mathrm{wal}_{k}(y) d x d y, \\
& \widehat{K}(-k, k)=\int_{0}^{1} \int_{0}^{1} K(x, y) \mathrm{wal}_{-k}(x) \overline{\operatorname{wal}_{k}(y)} d x d y=\int_{0}^{1} \int_{0}^{1} K(x, y) \overline{\operatorname{wal}_{k}(x) \mathrm{wal}_{k}(y)} d x d y .
\end{aligned}
$$

Since the kernel $K(x, y)$ is real we have $\widehat{K}(-k, k)=\widehat{K}(k,-k)$. Then by (45) we have

$$
\widehat{K}(-k, k)=\frac{1}{b^{2(n+1)}\left(e^{-(2 \pi / b) k_{n}}-1\right)^{2}}
$$

if $k_{n} \neq b / 2$. Since

$$
\frac{e^{-(2 \pi i / b) k_{n}}}{\left(e^{-(2 \pi i / b) k_{n}}-1\right)^{2}}=\frac{e^{(2 \pi i / b) k_{n}}}{\left(e^{(2 \pi i / b) k_{n}}-1\right)^{2}}
$$

we have (74).

The Theorem 8 follows.

REMARK 4. For the weighted Sobolev space with the reproducing kernel given by

$$
K(x, y)=1+\gamma\left(\frac{1}{2} B_{2}(\{x-y\})+\left(x-\frac{1}{2}\right)\left(y-\frac{1}{2}\right)\right)
$$

Dick and Pillichshammer [11; Theorem 10] proved that for every $\varepsilon>0$ there exists a digital net such that the mean square worst-case error is bounded by $O\left(1 / N^{2-\varepsilon}\right)$. But in $(62)$ the $\varepsilon$ does not occur.

\subsection{The mean square worst-case error for the weighted Sobolev space of order 2 .}

TheOREm 9. Let $b, \alpha$ and $\beta$ be integer numbers, $\alpha<\beta$, and

(i) $K(x, y)$ be a kernel of the form

$$
K(x, y)=1+\gamma B_{1}(x) B_{1}(y)+\frac{\gamma^{2}}{4} B_{2}(x) B_{2}(y)-\frac{\gamma^{2}}{24} B_{4}(|x-y|)
$$

(ii) $b=2, N=2^{\beta}$;

(iii) $x_{0}, x_{1}, \ldots, x_{N-1}$ be the van der Corput sequence in base $b=2$.

Then

$$
\begin{gathered}
\int_{0}^{1} \sup _{\substack{f \in H \\
\|f\| \leqslant 1}}\left|\frac{1}{N} \sum_{n=0}^{N-1} f\left(x_{n} \oplus \sigma\right)-\int_{0}^{1} f(x) d x\right|^{2} d \sigma=\frac{\gamma}{12 \cdot 2^{2 \beta}}+\frac{\gamma^{2}}{360 \cdot 2^{4 \beta}} ; \\
\int_{0}^{1} \sup _{\substack{f \in H \\
\|f\| \leqslant 1}}\left|\frac{1}{N} \sum_{n=0}^{N-1} f\left(\Phi\left(x_{n} \oplus \sigma\right)\right)-\int_{0}^{1} f(x) d x\right|^{2} d \sigma=\frac{\gamma}{12 \cdot 2^{2(\beta-\alpha)}}+\frac{\gamma^{2}}{360 \cdot 2^{4(\beta-\alpha)}}
\end{gathered}
$$


for $\Phi(x)=2^{\alpha} x \bmod 1$ and $\alpha<\beta$

$$
\int_{0}^{1} \sup _{\substack{f \in H \\\|f\| \leqslant 1}}\left|\frac{1}{N} \sum_{n=0}^{N-1} f\left(\Phi\left(x_{n} \oplus \sigma\right)\right)-\int_{0}^{1} f(x) d x\right|^{2} d \sigma=\frac{\gamma^{2}}{30 \cdot 2^{4 \beta}}
$$

for the tent transformation $\Phi(x)=\Phi_{0}(x)=1-|2 x-1|$.

Proof. In [1; p. 447] we have, for $k \geqslant 1,{ }^{15}$

$$
\widehat{K}(k, k)= \begin{cases}\frac{\gamma}{4 \cdot 2^{2(n+1)}}+\frac{\gamma^{2}}{120 \cdot 2^{4(n+1)}} & \text { if } k=2^{n}, \\ \frac{\gamma^{2}}{12 \cdot 2^{2(n+1+j+1)}}+\frac{\gamma^{2}}{20 \cdot 2^{4(n+1)}} & \text { if } k=k_{0}+k_{1} \cdot 2+\cdots+2^{j}+2^{n}, 0 \leqslant j<n,\end{cases}
$$

and for the tent transformation $\Phi_{0}(x)$

$$
\widehat{K}_{1}(k, k)= \begin{cases}\frac{\gamma}{4 \cdot 2^{2 n}}+\frac{\gamma^{2}}{120 \cdot 2^{4 n}} & \text { if } k=1+2^{n}, \\
\frac{\gamma^{2}}{12 \cdot 2^{2(n+j)}}+\frac{\gamma^{2}}{20 \cdot 2^{4 n}} & \text { if } k=k_{0}+k_{1} .2+\cdots+2^{j}+2^{n}, \\
0 & \begin{array}{r}
1 \leqslant j<n, 2 \mid\left(k_{0}+k_{1}+\cdots+k_{j-1}\right), \\
\text { otherwise. }
\end{array}\end{cases}
$$

Proof of (79). Again, for the van der Corput sequence $x_{n}, n=0,1, \ldots, N-1, N=2^{\beta}$, we have $($ see $(66))$

$$
\left|\frac{1}{N} \sum_{n=0}^{N-1} \mathrm{wal}_{k}\left(x_{n}\right)\right|^{2}= \begin{cases}0 & \text { if } 2^{\beta} \nmid k, \\ 1 & \text { if } 2^{\beta} \mid k,\end{cases}
$$

and by (10), in one-dimensional case, the left hand side of (79) is equal to

$$
\begin{aligned}
& \sum_{k=1}^{\infty} \widehat{K}(k, k)\left|\frac{1}{N} \sum_{n=0}^{N-1} \mathrm{wal}_{k}\left(x_{n}\right)\right|^{2} \\
& =\sum_{\substack{k=1 \\
2^{\beta} \mid k}}^{\infty} \widehat{K}(k, k)=\sum_{n=\beta}^{\infty} \widehat{K}\left(2^{n}, 2^{n}\right)+\sum_{\substack{n=\beta+1 \\
k=k_{\beta} 2^{\beta}+\cdots+2^{j}+2^{n} \\
\beta \leqslant j<n}}^{\infty} \widehat{K}(k, k) \\
& =\sum_{n=\beta}^{\infty}\left(\frac{\gamma}{4 \cdot 2^{2(n+1)}}+\frac{\gamma^{2}}{120 \cdot 2^{4(n+1)}}\right)+\sum_{n=\beta+1}^{\infty} \sum_{j=\beta}^{n-1} 2^{j-1-\beta+1}\left(\frac{\gamma^{2}}{12 \cdot 2^{2(n+1+j+1)}}+\frac{\gamma^{2}}{20 \cdot 2^{4(n+1)}}\right),
\end{aligned}
$$

which gives (79).

Proof of (82). By the tent transformation $\Phi_{0}(x)$ and by (12), the error (82) has the form

$$
\sum_{k=1}^{\infty} \widehat{K}_{1}(k, k)\left|\frac{1}{N} \sum_{n=0}^{N-1} \mathrm{wal}_{k}\left(x_{n}\right)\right|^{2}=\sum_{\substack{k=1 \\ 2^{\beta} \mid k}}^{\infty} \widehat{K}_{1}(k, k)
$$

\footnotetext{
${ }^{15}$ In this case $\widehat{K}(k,-k)=\widehat{K}(k, k)$ and $(83)$ and $(84)$ related by Theorem 5 .
} 


$$
\begin{aligned}
& =\widehat{K}_{1}\left(2^{\beta}, 2^{\beta}\right)+\widehat{K}_{1}\left(2^{\beta+1}, 2^{\beta+1}\right)+\widehat{K}_{1}\left(2^{\beta}+2^{\beta+1}, 2^{\beta}+2^{\beta+1}\right)+\sum_{n=\beta+2}^{\infty} \widehat{K}_{1}\left(2^{\beta}+2^{n}, 2^{\beta}+2^{n}\right) \\
& \quad+\sum_{n=\beta+2}^{\infty} \sum_{j=\beta}^{n-1} \widehat{K}_{1}\left(k_{\beta} 2^{\beta}+\cdots+2^{j}+2^{n}, k_{\beta} 2^{\beta}+\cdots+2^{j}+2^{n}\right) \\
& =0+0+\left(\frac{\gamma^{2}}{12 \cdot 2^{2(\beta+1+\beta)}}+\frac{\gamma^{2}}{20 \cdot 2^{4(\beta+1)}}\right)+\sum_{n=\beta+2}^{\infty}\left(\frac{\gamma^{2}}{12 \cdot 2^{2(n+\beta)}}+\frac{\gamma^{2}}{20 \cdot 2^{4 n}}\right) \\
& \quad+\sum_{n=\beta+2}^{\infty} \sum_{j=\beta+1}^{n-1} 2^{j-\beta-1}\left(\frac{\gamma^{2}}{12 \cdot 2^{2(n+j)}}+\frac{\gamma^{2}}{20 \cdot 2^{4 n)}}\right),
\end{aligned}
$$

which gives (82).

Proof of (80). We use (69) in the form

$$
\sum_{k=1,2^{\alpha} \mid k}^{\infty} \widehat{K}\left(\frac{k}{2^{\alpha}}, \frac{k}{2^{\alpha}}\right)\left|\frac{1}{N} \sum_{n=0}^{N-1} \operatorname{wal}_{k}\left(x_{n}\right)\right|^{2}=\sum_{k=1,2^{\beta} \mid k}^{\infty} \widehat{K}\left(\frac{k}{2^{\alpha}}, \frac{k}{2^{\alpha}}\right)=\sum_{k=1,2^{\beta-\alpha} \mid k}^{\infty} \widehat{K}(k, k) .
$$

Thus, Theorem 9 is proved.

REMARK 5. Thus we must take into the sum (85) also the terms $\widehat{K}\left(2^{n}, 2^{n}\right), \beta \leqslant n$, in which the sum has the order $O\left(N^{-2}\right)$. In (86) by (84) we do not take into account the terms $\widehat{K}_{1}\left(1+2^{n}, 1+2^{n}\right)$. This gives that the error (82) is better than the error (79) without special properties of the tent function $\Phi_{0}(x)$. The sums $(87)$ in the both cases are of the order $O\left(N^{-4}\right)$.

Finally, we also show that the result $O\left(N^{-4}\right)$ in (82) also depend on the number $N=2^{\beta}$ of terms $x_{0}, x_{1}, \ldots, x_{N-1}$. Naturally, $N$ can be selected arbitrary too, e.g.

$$
N=N_{0}+N_{1} b+N_{2} b^{2}+\cdots+N_{\beta-1} b^{\beta-1}+N_{\beta} b^{\beta}, \quad N_{\beta} \neq 0 .
$$

Then, for an arbitrary $k=k_{0}+k_{1} b+\cdots$, and the van der Corput sequence $x_{0}, x_{1}, \ldots, x_{N-1}$ we have

$$
\begin{aligned}
\sum_{n=0}^{N-1} \mathrm{wal}_{k}\left(x_{n}\right) & \\
= & \left(\sum_{n_{0}=0}^{b-1} e^{(2 \pi i / b) k_{0} n_{0}}\right) \cdots\left(\sum_{n_{\beta-1}=0}^{b-1} e^{(2 \pi i / b) k_{\beta-1} n_{\beta-1}}\right)\left(\sum_{n_{\beta}=0}^{N_{\beta}-1} e^{(2 \pi i / b) k_{\beta} n_{\beta}}\right) \\
& +\left(\sum_{n_{0}=0}^{b-1} e^{(2 \pi i / b) k_{0} n_{0}}\right) \cdots\left(\sum_{n_{\beta-1}=0}^{b-1} e^{(2 \pi i / b) k_{\beta-2} n_{\beta-2}}\right)\left(\sum_{n_{\beta}=0}^{N_{\beta-1}-1} e^{(2 \pi i / b) k_{\beta-1} n_{\beta-1}}\right) e^{(2 \pi i / b) k_{\beta} n_{\beta}} \\
& +\cdots+\left(\sum_{n_{0}=0}^{b-1} e^{(2 \pi i / b) k_{0} n_{0}}\right)\left(\sum_{n_{1}=0}^{N_{1}-1} e^{(2 \pi i / b) k_{1} n_{1}}\right) e^{(2 \pi i / b)\left(k_{2} N_{2}+\cdots+k_{\beta} N_{\beta}\right)} \\
& +\left(\sum_{n_{0}=0}^{b-1} e^{(2 \pi i / b) k_{0} n_{0}}\right) e^{(2 \pi i / b)\left(k_{1} N_{1}+k_{2} N_{2}+\cdots+k_{\beta} N_{\beta}\right)}
\end{aligned}
$$


where $n=n_{0}+n_{1} b+\cdots$ and $\left(\sum_{n_{\beta}=0}^{N_{j}-1} e^{(2 \pi i / b) k_{j} n_{j}}\right)=0$ if $N_{j}=0$. Putting $N=2^{\beta}+1$ in (88) we find

$$
\left|\frac{1}{N} \sum_{n=0}^{N-1} \mathrm{wal}_{k}\left(x_{n}\right)\right|^{2}= \begin{cases}\frac{1}{\left(2^{\beta}+1\right)^{2}} & \text { if } 2^{\beta} \nmid k, \\ 1 & \text { if } 2^{\beta} \mid k, k_{\beta}=0 \\ \left(\frac{2^{\beta}-1}{2^{\beta}+1}\right)^{2} & \text { if } 2^{\beta} \mid k, k_{\beta}=1 .\end{cases}
$$

For such $N=2^{\beta}+1$, summing $\sum_{k=1}^{\infty} \widehat{K}_{1}(k, k) \mid(1 / N) \sum_{n=0}^{N-1}$ wal $\left._{k}\left(x_{n}\right)\right|^{2}$ over $\widehat{K}_{1}(k, k)$ in $(84)$ we see that the mean square worst-case error has only the order $O\left(N^{-2}\right)$. In the following theorem we compute mean square worst-case error exactly dividing the sum $\sum_{n=0}^{b^{\beta}}$ wal $_{k}\left(x_{n}\right)$ into two parts $\sum_{n=0}^{b^{\beta}-1} \operatorname{wal}_{k}\left(x_{n}\right)$ and $\operatorname{wal}_{k}\left(x_{b^{\beta}}\right)$.

TheOREM 10. Let

(i) $K(x, y)$ be a kernel of the form

$$
K(x, y)=1+\gamma B_{1}(x) B_{1}(y)+\frac{\gamma^{2}}{4} B_{2}(x) B_{2}(y)-\frac{\gamma^{2}}{24} B_{4}(|x-y|)
$$

(ii) $b=2, N=2^{\beta}+1$;

(iii) $x_{0}, x_{1}, \ldots, x_{N-1}$ be the van der Corput sequence in base $b=2$.

Then for the tent transformation $\Phi_{0}(x)=1-|2 x-1|$ we have

$$
\begin{aligned}
& \int_{0}^{1} \sup _{\substack{f \in H \\
\|f\| \leqslant 1}}\left|\frac{1}{N} \sum_{n=0}^{N-1} f\left(\Phi_{0}\left(x_{n} \oplus \sigma\right)\right)-\int_{0}^{1} f(x) d x\right|^{2} d \sigma \\
& \quad=\left(\frac{2^{\beta}}{2^{\beta}+1}\right) \frac{\gamma^{2}}{30 \cdot 2^{4 \beta}}+\left(\frac{\gamma}{12}+\frac{\gamma^{2}}{360}\right) \frac{1}{\left(2^{\beta}+1\right)^{2}}+2 \frac{2^{\beta}}{\left(2^{\beta}+1\right)^{2}} \frac{\gamma^{2}}{2^{4 \beta}} \frac{7}{240}
\end{aligned}
$$

Proof. The following part holds for an arbitrary base $b$ and an arbitrary $k$. Divide

$$
\sum_{n=0}^{b^{\beta}} \operatorname{wal}_{k}\left(x_{n}\right)=\sum_{n=0}^{b^{\beta}-1} \operatorname{wal}_{k}\left(x_{n}\right)+\operatorname{wal}_{k}\left(x_{b^{\beta}}\right)
$$

then we have

$$
\begin{aligned}
\left|\sum_{n=0}^{b^{\beta}} \mathrm{wal}_{k}\left(x_{n}\right)\right|^{2}= & \left|\sum_{n=0}^{b^{\beta}-1} \mathrm{wal}_{k}\left(x_{n}\right)\right|^{2}+\mathrm{wal}_{k}\left(x_{b^{\beta}}\right) \overline{\mathrm{wal}_{k}\left(x_{b^{\beta}}\right)} \\
& +\left(\sum_{n=0}^{b^{\beta}-1} \mathrm{wal}_{k}\left(x_{n}\right)\right) \overline{\mathrm{wal}_{k}\left(x_{b^{\beta}}\right)}+\overline{\left(\sum_{n=0}^{b^{\beta}-1} \mathrm{wal}_{k}\left(x_{n}\right)\right)} \mathrm{wal}_{k}\left(x_{b^{\beta}}\right) .
\end{aligned}
$$

As we have proved

$$
\sum_{n=0}^{b^{\beta}-1} \operatorname{wal}_{k}\left(x_{n}\right)= \begin{cases}b^{\beta} & \text { if } b^{\beta} \mid k \\ 0 & \text { otherwise }\end{cases}
$$


and for $b^{\beta} \mid k$ we have $\operatorname{wal}_{k}\left(x_{b^{\beta}}\right)=e^{(2 \pi i / b) k_{\beta}}$. Inserting (90) in (12) we find

$$
\begin{aligned}
\sum_{k=1}^{\infty} \widehat{K}_{1}(k, k) \frac{1}{\left(b^{\beta}+1\right)^{2}}\left|\sum_{n=0}^{b^{\beta}} \mathrm{wal}_{k}\left(x_{n}\right)\right|^{2} \\
=\frac{1}{\left(b^{\beta}+1\right)^{2}} \sum_{k=1}^{\infty} \widehat{K}_{1}(k, k)\left|\sum_{n=0}^{b^{\beta}-1} \mathrm{wal}_{k}\left(x_{n}\right)\right|^{2} \\
+\frac{1}{\left(b^{\beta}+1\right)^{2}} \sum_{k=1}^{\infty} \widehat{K}_{1}(k, k) \\
+\frac{1}{\left(b^{\beta}+1\right)^{2}} \sum_{k=1}^{\infty} \widehat{K}_{1}(k, k)\left(\sum_{n=0}^{b^{\beta}-1} \mathrm{wal}_{k}\left(x_{n}\right)\right) 2 \cos \left(\frac{2 \pi i}{b} k_{\beta}\right) .
\end{aligned}
$$

Now, by (82) the term (91) is equal to (here $b=2$ )

$$
\left(\frac{b^{\beta}}{b^{\beta}+1}\right)^{2} \frac{\gamma^{2}}{30 \cdot 2^{4 \beta}}
$$

By Theorem 6 for the term (92) we have

$$
\sum_{k=1}^{\infty} \widehat{K}_{1}(k, k)=\int_{0}^{1} K(x, x) d x-\int_{0}^{1} \int_{0}^{1} K(x, y) d x d y
$$

and for Sobolev kernel we obtain

$$
\frac{\gamma}{12}+\frac{\gamma^{2}}{360}
$$

The final term (93) we express in the form

$$
2 \frac{b^{\beta}}{\left(b^{\beta}+1\right)^{2}}\left(\sum_{\substack{k=1 \\ k \in A}}^{\infty} \widehat{K}_{1}(k, k)-\sum_{\substack{k=1 \\ k \in B}}^{\infty} \widehat{K}_{1}(k, k)\right)
$$

where $A$ is the set of all $k$ satisfying

$$
\begin{gathered}
k=k_{0}+k_{1} \cdot 2+\cdots+2^{j}+2^{n}, \quad 1 \leqslant j<n, \\
2\left|\left(k_{0}+k_{1}+\cdots+k_{j-1}\right), \quad 2^{\beta}\right| k, \quad k_{\beta}=0,
\end{gathered}
$$

and $B$ is the set of all $k$ satisfying

$$
\begin{gathered}
k=k_{0}+k_{1} \cdot 2+\cdots+2^{j}+2^{n}, \quad 1 \leqslant j<n, \\
2\left|\left(k_{0}+k_{1}+\cdots+k_{j-1}\right), \quad 2^{\beta}\right| k, \quad k_{\beta}=1 .
\end{gathered}
$$

We find

$\sum_{\substack{k=1 \\ k \in A}}^{\infty} \widehat{K}_{1}(k, k)=\sum_{n=\beta+2}^{\infty}\left(\frac{\gamma^{2}}{12 \cdot 2^{2(n+\beta+1)}}+\frac{\gamma^{2}}{20 \cdot 2^{4 n}}\right)+\sum_{n=\beta+3}^{\infty} \sum_{j=\beta+2}^{n-1}\left(\frac{\gamma^{2}}{12 \cdot 2^{2(n+j)}}+\frac{\gamma^{2}}{20 \cdot 2^{4 n}}\right) \frac{2^{j-1-\beta}}{2}$

and

$$
\sum_{\substack{k=1 \\ k \in B}}^{\infty} \widehat{K}_{1}(k, k)=\sum_{n=\beta+1}^{\infty}\left(\frac{\gamma^{2}}{12 \cdot 2^{2(n+\beta)}}+\frac{\gamma^{2}}{20 \cdot 2^{4 n}}\right)+\sum_{n=\beta+3}^{\infty} \sum_{j=\beta+2}^{n-1}\left(\frac{\gamma^{2}}{12 \cdot 2^{2(n+j)}}+\frac{\gamma^{2}}{20 \cdot 2^{4 n}}\right) \frac{2^{j-1-\beta}}{2}
$$

which gives (94) in the form

$$
2 \frac{b^{\beta}}{\left(b^{\beta}+1\right)^{2}} \frac{\gamma^{2}}{2^{4 \beta}} \frac{7}{240}
$$




\section{Concluding remarks}

This section contains several remarks inspired by comments of referees. and

5.1. Classical method. The classical rectangular method of integration, for dimension $s=1$

$$
x_{n}=\frac{n+1 / 2}{N}, \quad n=0,1, \ldots, N-1,
$$

gives

$$
\begin{aligned}
& \left|\frac{1}{N} \sum_{n=0}^{N-1} f\left(x_{n}\right)-\int_{0}^{1} f(x) d x\right|^{2} \leqslant \frac{1}{12 N^{2}} \int_{0}^{1}\left(f^{\prime}(x)\right)^{2} d x, \\
& \left|\frac{1}{N} \sum_{n=0}^{N-1} f\left(x_{n}\right)-\int_{0}^{1} f(x) d x\right|^{2} \leqslant \frac{1}{320 N^{4}} \int_{0}^{1}\left(f^{\prime \prime}(x)\right)^{2} d x .
\end{aligned}
$$

It can be compared with (62) and (82) so that for van der Corput sequence $x_{0}, x_{1}, \ldots, x_{N-1}$ in base $b, N=b^{\beta}$, there exists $\sigma_{1} \in[0,1]$ such that for every $f \in H_{1},\|f\| \leqslant 1$, we have

$$
\left|\frac{1}{N} \sum_{n=0}^{N-1} f\left(x_{n} \oplus \sigma_{1}\right)-\int_{0}^{1} f(x) d x\right|^{2} \leqslant \frac{1}{6 N^{2}}
$$

and there exists $\sigma_{2} \in[0,1]$ such that for every $f \in H_{2},\|f\| \leqslant 1$, we have

$$
\left|\frac{1}{N} \sum_{n=0}^{N-1} f\left(\Phi_{0}\left(x_{n} \oplus \sigma_{2}\right)\right)-\int_{0}^{1} f(x) d x\right|^{2} \leqslant \frac{\gamma^{2}}{30 \cdot N^{4}}
$$

for the tent transformation $\Phi_{0}(x)=1-|2 x-1|$ and $b=2$. Here for $f \in H_{1}$ there exists $\int_{0}^{1}\left(f^{\prime}(x)\right)^{2} d x$ and for $f \in H_{2}$ there exists $\int_{0}^{1}\left(f^{\prime \prime}(x)\right)^{2} d x$.

If we construct a product rule for $s$ dimensions based on an $N$-point rule in one dimension, it requires $N^{s}$ points to achieve the same degree of accuracy (see Antia [12; p. 243]). Identifying $N^{s}$ as $N$, then from (95) we obtain $O\left(1 / N^{2 / s}\right)$ and from (96) we obtain $O\left(1 / N^{4 / s}\right)$. So, to get the errors $O\left(N^{-2+\varepsilon}\right)$ for $H_{1}, s>1$, and $O\left(N^{-4+\varepsilon}\right)$ for $H_{2}, s>1$, one has to consider complicated sequences $x_{n}$, e.g. lattices or digital nets (see [1], [2]).

Note that sequences $x_{n}, n=1,2, \ldots$, like van der Corput sequence have the advantage over sequences like $x_{n}=(n+1 / 2) / N$ : to estimate the integral using $N^{\prime}>N$ points, we do not need to re-calculate $f\left(x_{n}\right)$ for $n<N$. This and the following limit relation (97) is the basis of the quasi-Monte Carlo method (QMC).

5.2. quasi-Monte Carlo method. Define the $s$-dimensional step d.f. as

$$
F_{N}(\mathbf{x})=\frac{1}{N} \#\left\{0 \leqslant n \leqslant N: \mathbf{x}_{n} \in[\mathbf{0}, \mathbf{x})\right\}
$$

If $f:[0,1]^{s} \rightarrow \mathbb{R}$ is continuous, then the Riemann-Stieltjes integral gives

$$
\frac{1}{N} \sum_{n=1}^{N} f\left(\mathbf{x}_{n}\right)=\int_{[0,1]^{s}} f(\mathbf{x}) d F_{N}(\mathbf{x}) .
$$

Then Second Helly theorem (see [7; Theorem 4.1.0.11]) shows that the weak limit $F_{N}(\mathbf{x}) \rightarrow \mathbf{x}$ implies

$$
\int_{[0,1]^{s}} f(\mathbf{x}) d F_{N}(\mathbf{x}) \rightarrow \int_{[0,1]^{s}} f(\mathbf{x}) d \mathbf{x}
$$


for every continuous $f:[0,1]^{s} \rightarrow \mathbb{R}$. This is called Weyl's limit relation [7; Theorem 1.11.1.1]. For the rate of convergence of (97) it can be used.

Koksma-Hlawka's inequality [7; Theorem 1.11.3.1]: Let $f:[0,1]^{s} \rightarrow \mathbb{R}$ be of the bounded variation $V(f)$ in the sense of Hardy and Krause. Then for any sequence $\mathbf{x}_{0}, \ldots, \mathbf{x}_{N-1}$ in $[0,1)^{s}$ we have

$$
\left|\frac{1}{N} \sum_{n=0}^{N-1} f\left(\mathbf{x}_{n}\right)-\int_{[0,1]^{s}} f(\mathbf{x}) d \mathbf{x}\right| \leqslant V(f) D_{N}^{*}\left(\mathbf{x}_{n}\right),
$$

where the star discrepancy $D_{N}^{*}\left(\mathbf{x}_{n}\right)$ is defined by

$$
D_{N}^{*}\left(\mathbf{x}_{n}\right)=\sup _{\mathbf{x} \in[0,1)^{s}}\left|F_{N}(\mathbf{x})-\mathbf{x}\right| .
$$

The limit $D_{N}^{*}\left(\mathbf{x}_{n}\right) \rightarrow 0$ characterizes uniform distribution of the sequence $\mathbf{x}_{n}, n=0,1, \ldots$, in $[0,1)^{s}$.

5.3. $\boldsymbol{L}^{\mathbf{2}}$ discrepancy. a) The star-discrepancy $D_{N}^{*}$ in (98) can be replaced by $L^{2}$ discrepancy $D_{N}^{(2)}\left(\mathbf{x}_{n}\right)=\int_{[0,1]^{s}}\left(F_{N}(\mathbf{x})-\mathbf{x}\right)^{2} d \mathbf{x}$ expressed as [7; Item 1.11.4]

$$
D_{N}^{(2)}=\frac{1}{3^{s}}+\frac{1}{N^{2}} \sum_{m, n=1}^{N} \prod_{j=1}^{s}\left(1-\max \left(x_{m, j}, x_{n, j}\right)\right)-\frac{1}{2^{s-1} N} \sum_{n=1}^{N} \prod_{j=1}^{s}\left(1-x_{n, j}^{2}\right),
$$

where $\mathbf{x}_{n}=\left(x_{n, 1}, \ldots, x_{n, s}\right)$. Let $\mathbf{x}_{n}^{(1)}, n=0,1, \ldots, N-1$ minimize $D_{N}^{(2)}\left(\mathbf{x}_{n}\right)$. Let $\Phi(\mathbf{x})$ be a u.d.p. map and $\Phi\left(\mathbf{x}_{n}^{(2)}\right)=\mathbf{x}_{n}^{(1)}, n=0,1, \ldots, N-1$. Then

$$
D_{N}^{(2)}\left(\Phi\left(\mathbf{x}_{n}^{(2)}\right)\right) \leqslant D_{N}^{(2)}\left(\mathbf{x}_{n}^{(2)}\right), \quad D_{N}^{(2)}\left(\mathbf{x}_{n}^{(1)}\right) \leqslant D_{N}^{(2)}\left(\Phi\left(\mathbf{x}_{n}^{(1)}\right)\right) .
$$

Thus any u.d.p. map $\Phi(\mathbf{x})$ does not improve all sequences $\mathbf{x}_{n}$. For $s=1$ we have [7; Theorem 1.9.0.4]

$$
\left|\frac{1}{N} \sum_{n=1}^{N} f\left(x_{n}\right)-\int_{0}^{1} f(x) d x\right| \leqslant \sqrt{D_{N}^{(2)}} \sqrt{\int_{0}^{1}\left(f^{\prime}(x)\right)^{2} d x}
$$

and $D_{N}^{(2)}=\left(1 / N^{2}\right) \sum_{m, n=1}^{N} F_{0}\left(x_{m}, x_{n}\right)$, where

$$
F_{0}(x, y)=\frac{1}{3}+\frac{x^{2}+y^{2}}{2}-\frac{x+y}{2}-\frac{|x-y|}{2} .
$$

b) Hickernell [10] suggested, for Hilbert space integration, look at the square worst-case quasi-Monte Carlo error (6) as analogy of $L^{2}$ discrepancy:

$$
D_{N}^{K}=\int_{[0,1]^{2 s}} K(\mathbf{x}, \mathbf{y}) d \mathbf{x} d \mathbf{y}-\frac{2}{N} \sum_{n=0}^{N-1} \int_{[0,1]^{s}} K\left(\mathbf{x}_{n}, \mathbf{y}\right) d \mathbf{y}+\frac{1}{N^{2}} \sum_{n, m=0}^{N-1} K\left(\mathbf{x}_{m}, \mathbf{x}_{n}\right)
$$

and he found that for every $f \in H$ and $\mathbf{x}_{0}, \ldots, \mathbf{x}_{N-1}$ in $[0,1)^{s}$ we have

$$
\left|\frac{1}{N} \sum_{n=1}^{N} f\left(\mathbf{x}_{n}\right)-\int_{[0,1)^{s}} f(\mathbf{x})\right| \leqslant \sqrt{D_{N}^{K}} V_{K}(f),
$$

where $V_{K}(f)=\|f(\mathbf{x})-\mathbf{1}(f(\mathbf{x}) \cdot \mathbf{1}) /(\mathbf{1} \cdot \mathbf{1})\|$, see [10; p. 117]. The limit $D_{N}^{K} \rightarrow 0$ characterized uniformly distributed sequences in $[0,1)^{s}$, too. 
c) Let $W$ be a space of functions $f:[0,1]^{s} \rightarrow \mathbb{R}$ equipped with a measure of the Wiener type $d f$ and $\mathbf{x}_{n}, n=0,1, \ldots, N-1$, be a sequence in $[0,1)^{s}$. To characterize a rate of convergence

$$
\frac{1}{N} \sum_{n=0}^{N-1} f\left(\mathbf{x}_{n}\right) \rightarrow \int_{[0,1]^{s}} f(\mathbf{x}) d \mathbf{x}
$$

it can be used the integral

$$
\int_{W}\left|\frac{1}{N} \sum_{n=0}^{N-1} f\left(\mathbf{x}_{n}\right)-\int_{[0,1]^{s}} f(\mathbf{x}) d \mathbf{x}\right|^{2} d f .
$$

For $s=1$, Temirgaliev [13] proved that

$$
\int_{W}\left|\frac{1}{N} \sum_{n=0}^{N-1} f\left(\frac{n}{N}\right)-\int_{[0,1]^{s}} f(x) d x\right|^{2} d f=\left(\frac{\kappa}{12}+\frac{m^{2}}{16}\right) \frac{1}{N^{2}}+O\left(\frac{1}{N^{3}}\right) .
$$

Here he used: $F(x)$ is an infinitely divisible distribution function, $m=\int_{-\infty}^{+\infty} u d F(u), \kappa=$ $\int_{-\infty}^{+\infty} u^{2} d F(u)-m^{2}, W$ is a family of real valued functions $f(x)$ defined by Kolmogorov's theorem on the existence of stochastic processes and $d f$ is a measure on $W$ designed by $F(x)$. Methods of construction of $d f$ Temirgaliev discuses in [14], too.

5.4. Multivariate integration. In this example we shall see, again as in Theorem 10, that the tent map does not improve the following multidimensional sequence (99). Let $\mathbf{x}_{n}$ be an $s$-dimensional sequence,

$$
\mathbf{x}_{n}=\left(\frac{n_{1}}{b_{1}^{\beta_{1}}}, \frac{n_{2}}{b_{2}^{\beta_{2}}}, \ldots, \frac{n_{s}}{b_{s}^{\beta_{s}}}\right),
$$

where $n_{j}=0,1, \ldots, b_{j}^{\beta_{j}}-1, j=1,2, \ldots, s$. Let $K(\mathbf{x}, \mathbf{y})$ be a kernel of the type

$$
K(\mathbf{x}, \mathbf{y})=\prod_{j=1}^{s} K_{j}\left(x_{j}, y_{j}\right)
$$

where $\mathbf{x}=\left(x_{1}, x_{2}, \ldots, x_{s}\right)$ and $\mathbf{y}=\left(y_{1}, y_{2}, \ldots, y_{s}\right)$ and let $\Phi(\mathbf{x})$ be a u.d.p. map of the type

$$
\Phi(\mathbf{x})=\left(\Phi_{1}\left(x_{1}\right), \Phi_{2}\left(x_{2}\right), \ldots, \Phi_{s}\left(x_{s}\right)\right),
$$

where $\Phi_{j}(x)$ are one-dimensional u.d.p. maps. To short we denote $N_{j}=b_{j}^{\beta_{j}}$. Then the number of terms of (99) is $N=N_{1} N_{2} \cdots N_{s}$ and simplified $\mathbf{x}_{n}=\left(n_{1} / N_{1}, n_{2} / N_{2}, \ldots, n_{s} / N_{s}\right)$. By definition in Section 2 , for fixed $\mathbf{k}=\left(k_{1}, k_{2}, \ldots, k_{s}\right)$, we have

$$
\mathrm{wal}_{\mathbf{k}}(\mathbf{x})=\prod_{j=1}^{s} \mathrm{wal}_{k_{j}}\left(\frac{n_{j}}{N_{j}}\right)
$$

where the Walsh function wal $_{k_{j}}(x)$ is defined with respect to base $b_{j}$. Then

$$
\frac{1}{N} \sum_{n=0}^{N-1} \mathrm{wal}_{\mathbf{k}}\left(\mathbf{x}_{n}\right)=\prod_{j=1}^{s}\left(\frac{1}{N_{j}} \sum_{n_{j}=0}^{N_{j}-1} \mathrm{wal}_{k_{j}}\left(\frac{n_{j}}{N_{j}}\right)\right)
$$

and by means of one-dimensional result (66) we have

$$
\frac{1}{N} \sum_{n=0}^{N-1} \mathrm{wal}_{\mathbf{k}}\left(\mathbf{x}_{n}\right)= \begin{cases}0 & \text { if } \mathbf{b}^{\boldsymbol{\beta}} \nmid \mathbf{k} \\ 1 & \text { if } \mathbf{b}^{\boldsymbol{\beta}} \mid \mathbf{k} .\end{cases}
$$


Also

$$
\begin{aligned}
\widehat{K}_{1}(\mathbf{k}, \mathbf{k}) & =\int_{[0,1]^{2 s}} K(\Phi(\mathbf{x}), \Phi(\mathbf{y})) \mathrm{wal}_{\mathbf{k}}(\mathbf{x}) \overline{\mathrm{wal}_{\mathbf{k}}(\mathbf{y})} d \mathbf{x} d \mathbf{y} \\
& =\prod_{j=1}^{s} \int_{0}^{1} \int_{0}^{1} K_{j}\left(\Phi_{j}\left(x_{j}\right), \Phi_{j}\left(y_{j}\right)\right) \mathrm{wal}_{k_{j}}\left(x_{j}\right) \overline{\mathrm{wal}_{k_{j}}\left(y_{j}\right)} d x_{j} d y_{j}=\prod_{j=1}^{s} K_{1, j}\left(k_{j}, k_{j}\right) .
\end{aligned}
$$

Applying (100) in Theorem 3 we find

$$
\begin{aligned}
\int_{[0,1]^{s}} & \sup _{\substack{f \in H \\
\|f\| \leqslant 1}}\left|\frac{1}{N} \sum_{n=0}^{N-1} f\left(\Phi\left(\mathbf{x}_{n} \oplus \boldsymbol{\sigma}\right)\right)-\int_{[0,1]^{s}} f(\mathbf{x}) d \mathbf{x}\right|^{2} d \boldsymbol{\sigma} \\
= & \sum_{\substack{\mathbf{k} \in \mathbb{N}_{0}^{s} \\
\mathbf{k} \neq \mathbf{0}, \mathbf{b}^{\boldsymbol{\beta}} \mid \mathbf{k}}} \widehat{K}_{1}(\mathbf{k}, \mathbf{k})=\prod_{j=1}^{s}\left(\sum_{\substack{k_{j}=0 \\
b_{j}^{\beta_{j}} \mid k_{j}}}^{\infty} \widehat{K}_{1, j}\left(k_{j}, k_{j}\right)\right)-\widehat{K}_{1}(\mathbf{0}, \mathbf{0}) \\
= & \prod_{j=1}^{s}\left(\sum_{k_{j}=1}^{\infty} \widehat{K}_{1, j}\left(k_{j}, k_{j}\right)+\widehat{K}_{j}(0,0)\right)-\prod_{j=1}^{s} \widehat{K}_{j}(0,0) \\
= & \prod_{j=1}^{s}\left(\int_{0}^{b_{j}^{\beta_{j}} \mid k_{j}} \sup _{\| \in H}\left|\frac{1}{N_{j}} \sum_{n_{j}=0}^{N_{j}-1} f\left(\Phi_{j}\left(\frac{n_{j}}{N_{j}} \oplus \sigma\right)\right)-\int_{0}^{1} f(x) d x\right|^{2} d \sigma+\int_{0}^{1} \int_{0}^{1} K_{j}(x, y) d x d y\right) \\
& -\int_{[0,1]^{2 s}} K(\mathbf{x}, \mathbf{y}) d \mathbf{x} d \mathbf{y}
\end{aligned}
$$

because

$$
\widehat{K}_{1}(\mathbf{0}, \mathbf{0})=\int_{[0,1]^{2 s}} K(\Phi(\mathbf{x}), \Phi(\mathbf{y})) d \mathbf{x} d \mathbf{y}=\int_{[0,1]^{2 s}} K(\mathbf{x}, \mathbf{y}) d \mathbf{x} d \mathbf{y}=\widehat{K}(\mathbf{0}, \mathbf{0})
$$

Now, putting

$$
\begin{gathered}
b_{1}=b_{2}=\cdots=b_{s}=2, \quad \beta_{1}=\cdots=\beta_{s}=\beta, \quad N=\left(2^{\beta}\right)^{s}, \\
K_{j}(x, y)=K(x, y)=1+\gamma B_{1}(x) B_{1}(y)+\frac{\gamma^{2}}{4} B_{2}(x) B_{2}(y)-\frac{\gamma^{2}}{24} B_{4}(|x-y|),
\end{gathered}
$$

and using the $s$-dimensional tent u.d.p. map

$$
\Phi(x)=\Phi_{0}(\mathbf{x})=\left(1-\left|2 x_{1}-1\right|, 1-\left|2 x_{2}-1\right|, \ldots, 1-\left|2 x_{s}-1\right|\right),
$$

and inserting (82) to (101), also using $\int_{0}^{1} \int_{0}^{1} K(x, y) d x d y=1$, we find the $s$-dimensional mean square worst-case error (101) as

$$
\left(\frac{\gamma^{2}}{30 \cdot 2^{4 \beta}}+1\right)^{s}-1
$$

Thus, for the sequence (99) and applying the tent u.d.p. map $\Phi_{0}(\mathbf{x})$, we obtain the error $O\left(1 / N^{4 / s}\right)$ what is contrary to the [1], where in [1; Theorem 5] is proved that for every $\varepsilon>0$ there exists a digital net such that the mean square worst-case error is bounded by $O\left(1 / N^{4-\varepsilon}\right)$.

We thank the anonymous referees for their many helpful suggestions. 


\section{Bibliography}

[1] L. L. Cristea, J. Dick, G. Leobacher, F. Pillichshammer, "The tent transformation can improve the convergence rate of quasi-Monte Carlo algorithms using digital nets", Numer. Math., 105:3 (2007), 413-455.

[2] F. J. Hickernell, "Obtaining $O\left(N^{-2+\varepsilon}\right)$ convergence for lattice quadrature rules", Monte Carlo and Quasi-Monte Carlo Methods, 2000, Springer-Verlag, Berlin, 2002, 274-289.

[3] G. Larcher, "A class of low-discrepancy point-sets and its applications to numerical integration by number-theoretical methods", Österreichisch-Ungarisch-Slowakisches Kolloquium über Zahlentheorie, Grazer Math. Ber., 318, Karl-Franzens-Univ. Graz, Graz, 1993, 69-80.

[4] E. Novak, H. Woźniakowski, Tractability of Multivariate Problems. Vol. II. Standard Information for Functionals, EMS Tracts Math., 12, European Math. Soc., Zürich, 2010.

[5] J. Dick, F. Pillichshammer, Digital Nets and Sequences. Discrepancy Theory and Quasi-Monte Carlo Integration, Cambridge Univ. Press, Cambridge, 2010.

[6] O. Strauch, Unsolved Problems, http://www.boku.ac.at/MATH/udt/unsolvedproblems.pdf.

[7] O. Strauch, Š. Porubský, Distribution of Sequences: A Sampler, Schr. Slowak. Akad. Wiss., 1, Peter Lang, Frankfurt am Main, 2005.

[8] S. Steinerberger, "Uniform distribution preserving mappings and variational problems", Unif. Distrib. Theory, 4:1 (2009), 117-145.

[9] I. H. Sloan, H. Woźniakowski, "When are quasi-Monte Carlo algorithms efficient for hig dimensional integrals?", J. Complexity, 14:1 (1998), 1-33.

[10] F. J. Hickernell, "Lattice rules: How well do they meaure up?", Random and Quasi-Random Point Sets, Lecture Notes in Statist., 138, Springer-Verlag, New York, 1998, 109-166.

[11] J. Dick, F. Pillichshammer, "Multivariate integration in weighted Hilbert space based on Walsh functions and weighted Sobolev spaces", J. Complexity, 21:2 (2005), 149-195.

[12] H. M. Antia, Numerical Methods for Scientists and Engineers, Birkhäuser Verlag, Basel, 2002.

[13] N. Temirgaliev, "On an application of infinitely divisible distributions to quadrature problems", Anal. Math., 14:3 (1988), 253-258.

[14] Н. Т. Темиргалиев, "О построении вероятностных мер на функциональных классах", Аналитическая теория чисел и приложения, Сборник статей. К 60-летию со дня рождения профессора Анатолия Алексеевича Карацубы, Тр. МИАН, 218, Наука, М., 1997, 397-402; N. T. Temirgaliev, "On the construction of probability measures of functional classes", Proc. Steklov Inst. Math., 218 (1997), 396-401. 